
\title{
3 Research Square \\ The Molecular Mechanisms of Suanzaoren Prescription for Anti-Anxiety Were Investigated Based on Network Pharmacology
}

\section{Xiaohong Bao}

Shandong University of Traditional Chinese Medicine

Tianyuan Ye

Shandong University of Traditional Chinese Medicine

\section{Xiaolong Wang}

Shandong University of Traditional Chinese Medicine

\section{Lu Han}

Beijing Institute of Pharmacology and Toxicology, State Key Laboratory of Toxicology and Medical Countermeasures

\section{Tongxing Wang}

GeneNet Pharmaceuticals Co. Ltd., Tianjin

\section{Dongmei Qi}

Shandong University of Traditional Chinese Medicine

\section{Xin Wang}

Shandong University of Traditional Chinese Medicine

Xiaorui Cheng ( $\nabla$ cxr916@163.com)

Shandong University of Traditional Chinese Medicine

\section{Research Article}

Keywords: molecular mechanisms, Suanzaoren prescription, anti-anxiety, investigate, network, psychological and environmental factors, disorders

Posted Date: July 20th, 2021

DOl: https://doi.org/10.21203/rs.3.rs-719079/v1

License: (c) (1) This work is licensed under a Creative Commons Attribution 4.0 International License. Read Full License 


\section{Abstract}

Anxiety is a common, universal disease caused by psychological and environmental factors. There are medications available to treat anxiety disorders, but these are accompanied by problems such as addiction and withdrawal difficulties. Suanzaoren Prescription (SZRP) is often used to treat anxiety disorders in traditional Chinese medicine clinical practice. However, its therapeutic mechanism remains unclear.

This work aims to identify potential core therapeutic targets and predict major bioactive compounds by means of network pharmacology, screen compounds that may bind to anxiety targets by molecular docking methods, and validate and evaluate possible therapeutic mechanisms of compounds for anxiety in vitro cell experiments.

Datebase TCMSP, BATMAN-TCM, ETCM, TCMGeneDIT and TCMID was used to obtain SZRP compounds, while TCMSP, BATMAN-TCM and ETCM were used to obtain target of compounds. The targets of anxiety disorders were obtained through database TTD, OMIM, PharmgKB, CTD, DrugBank and Metacore. The network diagram is drawn by Cytoscape 3.7.2 software and STRING. Biological process and KEGG pathway analysis were conducted by MetaCore and NIMNT. AutoDock Vina software was used to conduct molecular docking. The calcium Flux assay was employed to detect the activation of compounds on CNR1 in cell line CHO-K1/CNR1/Ga15.

The 545 compounds in SZRP were collected. We found there were 1050 potential targets of these compounds in SZRP which involved in brain disease, mood disorder, endocrine system disease, bipolar disorder, etc. There were 117 potential targets of SZRP against anxiety (such as DRD2, BDNF, COMT, SLC6A4, HTR1A, etc.), which played the roles in regulation of membrane potential and synaptic transmission, nicotine addiction, retinol metabolism, GABAergic and serotonergic synapse, etc. The results of molecular docking indicated there were the biggest affinity between Jujuboside B and DRD2, BDNF, COMT, SLC6A4, Anemarsaponin B_qt and HTR1A, n-methylasimilobine and MAPK3. Based on cell $\mathrm{CHO}-\mathrm{K} 1 / \mathrm{CNR} 1 / \mathrm{Ga} 15$ and calcium flux technique, we found Chrysophanol, Kaempferol and Emodin have

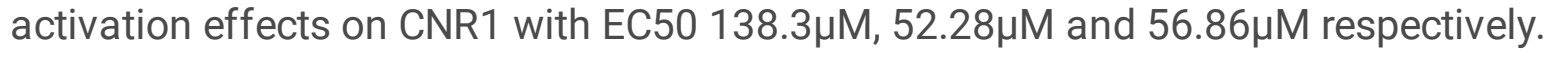

These results indicated that the effects of SZRP against anxiety were correlated with synaptic transmission, substance addiction and retinol metabolism, especially targeted on CNR1. It suggested that the therapeutic mechanism of SZRP treating anxiety might be due to the weak action of multiple compounds on multiple pathways and biological processes.

\section{Introduction}

Anxiety disorder is one of the most common mental disorders 1. It was reported that the prevalence of 12month Diagnostic and Statistical Manual of Mental Disorders (DSM-IV) anxiety disorder was $9.8 \%$ in 21 countries 2. And lifetime prevalence of 7.57 percent and 12-month prevalence of 4.98 percent only in China 3. It is speculated that the number of anxiety patients is also on the rise year by year with the 
increase of social pressure. The benzodiazepines with sedative effects were generally used in clinical treatment. For example, diazepam quickly exert anxiolytic effects by enhancing GABAergic neurotransmission, which advantages of these drugs are rapid onset 4,5 . However, due to poor tolerance of these drugs, long-term use in large quantities presented drug dependence 6 and sudden withdrawal of drugs produced withdrawal reactions, such as inattention, depression, insomnia and other symptoms 7. Excessive use could cause poisoning, manifested as unconsciousness, coma and other symptoms 8. Therefore, there is no ideal drug for the treatment of anxiety.

Suanzaoren Prescription (SZRP) is often used to treat anxiety disorders in traditional Chinese medicine (TCM) clinical practice. SZRP also named Suanzaoren decoction, Ziziphi Spinosae Semen, Zizyphus Combination, Suanzaoren Formulae, Suan Zao Ren Tang. SZRP comes from the "Synopsis of Golden Chamber "( Jinkui Yaolue, the Eastern Han Dynasty, Zhang Zhong-jing). SZRP was consists of Ziziphus Jujuba Var. Spinosa, Poria cocos Wolf, Anemarrhenae Rhizoma, Ligusticum chuanxiong Hort., Glycyrrhiza.glabra L. on 15:6:6:6:3 (Table 1).

Mounts of clinical studies have shown that SZRP has certain anti-anxiety effects and had no toxic side effects. A clinical study reported that 60 generalized anxiety disorder (GAD) patients (mean age 32) were randomly divided into SZRP group and control group with 30 patients in each one. After the treatment of SZRP, the Hamilton Anxiety Scale (HAMA) and Self-Rating Anxiety Scale (SAS) scores were significantly lower than those of control group 9. Another clinical study reported that $105 \mathrm{GAD}$ in the elderly patients were randomly divided into treatment group (treatment with SZRP and paroxetine, mean age 72) with 52 patients and control group (treatment with paroxetine, mean age 69) with 53 patients. After the treatment of SZRP combined with paroxetine for 1 weeks $\bigotimes$ HAMA scores were clearly lower than control group, and after 6 weeks of treatment, there was no significant difference in HAMA score. However, the incidence of adverse reactions in the treatment group was significantly lower than that in the control group 10.

Additional, the administration of SZRP increased open arm entry (OE) and open arm time (OT) of anxiety rat model induced by elevated plus maze (EPM) 11-14. Studies indicated that the treatment of SZRP regulated the levels of neuropeptide $Y 15,5$-HIAA, GABA, GABA/ Glu, NE 11, 16, 17 , $\beta$-endorphin ( $\beta$-EP) 14, increased the contents of adreno-cortico-tropic-hormone (ACTH), IL-1 $\beta$ and TNF- $\alpha$ in serum 18. Furthermore, our previous study showed that Spinosin, Jujuboside BI, Ononin and Timosaponin El in SZRP were easy to enter the blood, especially, Spinosin, Timosaponin B-II and Ononin were found to be chemical components that could easily cross the blood-brain barrier and enter cerebrospinal fluid 17. However, it remains unclear for the therapeutic mechanism of SZRP treating anxiety disorders.

In order to investigate mechanism of SZRP for anti-anxiety, we collected compounds in SZRP and searched their potential targets to explore the main diseases treated by SZRP. Secondly, the shared targets of compounds in SZRP and anxiety were achieved, their biological process and pathways were enriched and analyzed. Then the molecular docking was employed to predict the affinity between compounds in SZRP and targets with related to anxiety. Finally, based on cell CHO-K1/CNR1/Ga15 and calcium flux technique, we evaluated the activity of some compounds targeting on CNR1. 


\section{Materials And Methods}

The whole workflow was illustrated in Fig. 1. First, known compounds in SZRP were collected from TCMSP, BATMAN-TCM, ETCM, TCMGenenDIT and TCMID databases. TCMSP, BATMAN-TCM and ETCM were used to obtain potential targets of compounds and conduct enrichment analysis by NIMNT and MetaCore. Secondly, the related targets of anxiety were obtained from MetaCore, OMIM, PhamGkb, TTD, CTD and Durgbank. Then the common targets of SZRP and anxiety were enriched for analysis employing NIMNT and MetaCore databases. Finally, AutoDock Vina were used to predict the compound-target group by molecular docking, and the activity of compounds was detected by the calcium flux assay.

\section{Identification of known compounds in Suanzaoren prescription}

All compounds in the five herbs of SZRP were collected from TCMSP ( https://lsp.nwu.edu.cn/ ), BATMAN-TCM (http://bionet.ncpsb.org.cn/batman-tcm/), ETCM (http://www.tcmip.cn/ETCM/index.php/Home/), TCMGeneDIT (http://tcm.lifescience.ntu.edu.tw/HanMedDBsearch.html), TCMID (http://119.3.41.228:8000/tcmid/).

\section{Identification of potential targets associated with Suanzaoren prescription and anxiety}

Obtaining the potential targets of SZRP using TCMSP, ETCM and BATMAN-TCM. Targets related to anxiety were derived from six databases, TTD ( https://ttd.org/), OMIM (https://omim.org/), PharmGkb (https://www.pharmgkb.org/), CTD (http://ctd.mdibl.org/), DRUGBANK (https://go.drugbank.com/) and MetaCore (https://portal.genego.com/). And the protein targets derived from TCMSP and TTD were transformed into genes using STRING version 11.0 (https://string-db.org/).

\section{Construction of networks}

Three networks were constructed by employing the network visualization software Cytoscape 3.7.2. These networks were herb-compound network ( $\mathrm{H}-\mathrm{C}$ network), compound-targets network (C-T network) and core potential targets of SZRP against anxiety. Isolated nodes do not appear in the external diagram.

The possible inter-protein interactions (PPIs) were derived from STRING database, which covered almost all functional interactions between the expressed proteins. And PPI network pairs with overall combined scores above 0.4 were selected and the isolated nodes were not shown. The PPI network results of STRING were output in TSV format, and then imported into Cytoscape 3.2.1 to screen the core potential targets.

\section{Functional Annotation and Enrichment Analysis}

Statistical analysis of functional annotation of biological process, KEGG pathway enrichment and disease enrichment analysis of the potential target for SZRP was performed by MetaCore and NIMNT (http://www.idrug.net.cn/NIMNT/). Biological process and KEGG pathway analysis were conducted for 
the common potential targets of SZRP and anxiety by MetaCore and NIMNT. Selection of enrichment results was statistically significant (Pvalue区0.05) and rank top 50 terms.

\section{Molecular docking simulation}

In order to study the active components of SZRP for the prevention and treatment of anxiety and to analyze its possible targets, this study used software AutoDock Vina for molecular docking analysis. The conformation sampling adopts the default optimization parameter, executes each operation in singlethread mode, and adopts the default scoring function using AutoDock Vina. The compounds with molecular weight < 500Da in SZRP were selected for docking using AutoDock Vina. Based on our previous studies on SZRP, we selected the core target of SZRP anti-anxiety and compounds into blood and brain for docking using AutoDock Vina.

\section{Determination of compound activity}

We selected five compounds in SZRP, Isomangiferin (B21543), Liquiritigenin (B20416), Kaempferol (B21126), Chrysophanol (B20238) and Emodin (B20240) (The above compounds were purchased from shanghai yuanye Bio-Technology Co., Ltd, HPLC $\geq 98 \%$ ) to detect the effect on CNR1 receptor with calcium flux method. The positive compound was CP-55940 (Sigma, C1112). Compound was dissolved in HBSS (Gibco, 14175-095) buffer [including 20 mM HEPES (Gibco, 156030-080 2042335) pH 7.4] and diluted with DMSO (SIGMA-ALDRICH, D2650). The cell line CHO-K1/CNR1/Ga15 expressing CNR1 receptor (GenScript, M00299, R10011904-2) was used to investigate the activity of compound on CNR1 receptor. The cell CHO-K1/CNR1/Ga15 was cultured in medium Ham's F12『Gibco, R8142) (added 10\% FBS, $100 \mu \mathrm{g} / \mathrm{ml}$ HygromycinB, $200 \mu \mathrm{g} / \mathrm{ml}$ Zeocin), and incubator with $37^{\circ} \mathrm{C}, 5 \% \mathrm{CO}_{2}$. The CHO$\mathrm{K} 1 / \mathrm{CNR} 1 / \mathrm{Ga} 15$ cells were seeded in a 384-well black-wall (Corning, 3764), clear-bottom plate (Corning, 3656 ) at a density of 10,000 cell per well in $20 \mu \mathrm{L}$ growth medium at 18 hours prior to the day of calcium assay and maintained at $37{ }^{\circ} \mathrm{C} / 5 \% \mathrm{CO}_{2}$. For agonist assay, $20 \mu \mathrm{L}$ dye-loading solution (Ham's $\mathrm{F} 12,10 \%$ FBS) was added into the wells. Then the plate was placed into a $37^{\circ} \mathrm{C}$ incubator for 60 minutes, followed by a 15 minutes' incubation (Thermo, 3111) at room temperature. At last, $10 \mu \mathrm{L}$ compound was added into well of the assay plate during reading in FLIPR tetra (Moleacular Devices). For FLIPR reading, the plate containing $5 \times$ compound solution was placed in FLIPR. Solutions were added into the cell plate automatically at the 20 seconds and the fluorescence signal was monitored for an additional 100 seconds (21 sec to $120 \mathrm{sec}$.). Data were recorded by ScreenWorks (version 3.1) as FMD files with FLIPR. Data acquisition and analyses was performed using ScreenWorks (version 3.1) program and exported to Excel. The average value of the first 20 seconds' reading was calculated as the baseline and the relative fluorescent units ( $\triangle \mathrm{RFU})$ intensity values were calculated by subtracting the average value of baseline from the maximal fluorescent units (21s to 120 s).

The \% stimulation was calculated with the following equation: \% Stimulation $=(\triangle \mathrm{RFUCompound}-$ $\triangle \mathrm{RFUBackground}) /(\triangle \mathrm{RFUAgonist}$ control - $\triangle \mathrm{RFUBackground}) * 100 \%$. 
Dose response curves were fitted with four-parameter-logistic-equation by the software GraphPad Prism 8. The four parameters logistic equation was: Y=Bottom + (T op-Bottom)/ $\left(1+10^{\wedge}((\right.$ LogEC50$\mathrm{X})^{\star}$ HillSlope))

$\mathrm{X}$ is the logarithm of concentration. $\mathrm{Y}$ is the response.

\section{Results}

In order to collect compounds in SZRP and potential targets of SZRP as much as possible, we used the Chinese name, botanical name and common name of herbs in SZRP to search databases and literatures (Table1).

\section{Ingredients in Suanzaoren prescription}

Based on TCMSP, BATMAN-TCM, ETCM, TCMGenenDIT and TCMID databases, we collected 64 compounds in Ziziphi Spinosae Semen (Suanzaoren), 103 compounds in Poria Cocos Wolf. (Fuling), 123 compounds in Anemarrhenae Rhizoma (Zhimu), 292 compounds in Ligusticum chuanxiong Hort. (Chuanxiong), and 28 compounds in Glycyrrhiza.glabra L. (Zhigancao). Combining the components in the five herbs and removing the duplicate components of the same name, a total of 545 compounds in the SZRP were obtained (Table 2) (Additional file1).

We established the herb-compound network for SZRP (Fig. 2) (Additional file 2). This herbal-compound network is made up of 5 herbs and 545 compounds. This network had 550 nodes and 610 edges (herbcompound). This $\mathrm{H}-\mathrm{C}$ network had the average shortest path length 3.08, average degree 2.22 and average neighborhood connectivity 196.43 .

\section{Potential targets of Suanzaoren prescription}

The 305 potential target proteins were obtained from the database TCMSP, after mapped using the STRING database and removed the duplicates, there were 111 potential gene targets for SZRP. For each component in herbs, database BATMAN-TCM ranks its predicted candidate targets based on the order of the reduced scores given by the target-predictive algorithm for herb-target interaction prediction. There were potential 847 gene targets for SZRP with gene score $₫ 20$ from BATMAN-TCM. We collected 408 potential target genes for the compounds in SZRP from database ETCM. The potential targets of 330 compounds were found. Combining targets of these 3 databases, SZRP had a total of 1050 potential targets were obtained after eliminating duplicates (Table 3) (Additional file 3).

A network of compound-targets ( $\mathrm{H}-\mathrm{T}$ network) for SZRP was constructed (Fig. 3). This C-T network had 1380 nodes (330 compound nodes and 1050 target nodes) and 6423 edges. This $\mathrm{H}-\mathrm{T}$ network had the average shortest path length 3.88, average closeness is 0.07 and average degree 9.31 (Additional file 4). Among them, compounds with the degree 3 included FER, N-Trans-Feruloyltyramine, 6-O-E-Feruloylajugol, B-Sitosterol-B-D-Glucoside, palmitic acid, Choline, Chrysanthemaxanthin. There were 51 compounds with the degree 2, for example mairin, ceanothic acid, Jujuboside A and Jujuboside B, etc 


\section{Disease enrichment analysis potentially treated by Suanzaoren prescription}

The disease enrichment analysis of 1050 potential targets of SZRP were performed using NIMNT and MetaCore databases in order to investigate disease potentially treated by SZRP.

NIMNT-based enrichment analysis showed that the top 10 ( $P$ value $<0.001$ and from small to large) diseases potentially treated by SZRP included brain disease, Alzheimer's disease, tauopathy, migraine, nutrition disease, pre-eclampsia, coronary artery disease, overnutrition, mood disorder, obesity (Fig. 4) (Additional file 5).

The results based on MetaCore database showed the top 10 ( $P$ value $<0.001$ and from small to large) diseases potentially treated by SZRP were chemically-induced disorders; pathological conditions, signs and symptoms; mental disorders; nutritional and metabolic diseases; psychiatry and psychology; metabolic diseases; movement disorders; genetic diseases, inborn; congenital, hereditary, and neonatal diseases and abnormalities; bipolar and related disorders (Fig. 4) (Additional file 5).

We conducted a comparative analysis of the top 50 diseases potentially treated by SZRP based on databases NIMNT and MetaCore. There were 11 diseases with overlap (Table 4). They were brain disease, mood disorder, endocrine system disease, bipolar disorder, etc.

Anxiety disorders, the most prevalent mental or mood disorders, is considered a polygenic, multifactorial trait wherein the continuum of physiological anxiety up to psychopathology is likely to be shaped by the interplay of central nervous system, endocrine system and immune system. This disease enrichment analysis suggested that SZRP may be used to treat anxiety.

\section{Targets related to anxiety}

We collected targets related to anxiety from six database (Table 5). A total of 67 gene targets related to anxiety were found in OMIM. 7 gene targets for anxiety were found in PharmGkb. The 41 therapeutic targets for anxiety disorders from TTD were converted by STRING. The 50 genetic targets for anxiety disorders were found in CTD. Durgbank has 73 protein targets for anxiety disorders, which are converted to 73 by STRING. The 133 genes associated with anxiety were found in MetaCore. After integrating targets from the six databases and removing duplicates, there were 249 targets related to anxiety (Table 5) (Additional file 6).

We collected 1050 potential targets for compounds in SZRP and 249 targets associated with anxiety disorders. There were 117 targets after overlap between these types of targets (Table 6) (Additional file 7).

We constructed the PPI network related to SZRP for anti-anxiety employing 117 common potential targets (Fig. 5) (Additional file 8). This PPI network had the average shortest path length 2.06, average degree 23.03 and average neighborhood connectivity 29.24. The target with the highest degree is DRD2, whose degree was 54. Through the double median of Betweenness, Closeness and Degree three 
parameters, 11 core targets were obtained after four screening. The 5 targets were ranged from the largest to the smallest in order of Degree value, and were DRD2, BDNF, COMT, SLC6A4, HTR1A.

\section{Functional annotation of biological process}

The results of NIMNT-based GO-BP enrichment analysis showed that the biological processes of the top 10 ( $P$ value $<0.05$ and from small to large) included, regulation of postsynaptic membrane potential, regulation of membrane potential, monoamine transport, $G$ protein-coupled receptor signaling pathway coupled to cyclic nucleotide second messenger, response to ammonium ion, response to xenobiotic stimulus, modulation of chemical synaptic transmission, regulation of trans-synaptic signaling, catecholamine transport, regulation of amine transport (Fig. 6) (Additional file 9).

The enrichment analysis results of Process Networks based on MetaCore showed that the biological processes of the top 10 ( $P$ value $<0.05$, arranged from small to large) (Fig. 6) (Additional file 9 ) include, chemical synaptic transmission, anterograde trans-synaptic signaling, trans-synaptic signaling, synaptic signaling, regulation of postsynaptic membrane potential, cell-cell signaling, regulation of membrane potential, response to organic cyclic compound, system process, regulation of biological quality.

After comparative analysis of the biological processes of the top $20(P$ value $<0.05$, arranged from small to large) in the two databases, there is 3 biological process after taking the intersection. They were regulation of postsynaptic membrane potential, regulation of membrane potential, and synaptic transmission, GABAergic.

\section{Enrichment analysis of KEGG pathway}

NIMNT-based KEGG enrichment analysis results showed that the top 10 pathways ( $P$ value $<0.05$, arranged from small to large) included, neuroactive ligand-receptor interaction, nicotine addiction, serotonergic synapse, morphine addiction, GABAergic synapse, drug metabolism-cytochrome P450, retrograde endocannabinoid signaling, chemical carcinogenesis, calcium signaling pathway, taste transduction (Fig. 7) (Additional file 10).

MetaCore based Pathway enrichment analysis results showed that the top 10 ( $P$ value $<0.05$, arranged from small to large) (Fig. 7) (Additional file 10) pathways included, tinnitus-associated changes in auditory pathway, protein folding and maturation_insulin processing, mediated direct regulation of xenobiotic metabolizing enzymes, mediated direct regulation of xenobiotic metabolizing enzymes, mediated direct regulation of xenobiotic metabolizing enzymes, mediated direct regulation of xenobiotic metabolizing enzymes, nicotine signaling (general schema), retinol metabolism, estradiol metabolism, serotonin modulation of dopamine release in nicotine addiction.

There were 4 pathways in total after taking the intersection of the top 20 ( $P$ value $<0.05$, arranged from small to large) pathways in the two databases. The common pathways were nicotine addiction, retinol metabolism, GABAergic and serotonergic synapse. 


\section{Molecular docking simulation}

According to our previous studies, the components of SZRP into blood and brain were Spinosin, Ononin, Timosaponin El, Timosaponin B-II and Jujuboside BI 17. According to the chemical structure of these 5 compounds, there were other 7 compounds with the same skeleton structure in SZRP. Among these 12 compounds, namely Anemarsaponin B, Anemarsaponin B_qt, Anemarsaponin E, Anemarsaponin E_qt, Jujuboside B, Jujuboside B_qt, ononin, Spinosin, Timosaponin B III, Timosaponin B III_qt, Timosaponin BII, Timosaponin BII_qt. We performed molecular docking analysis of 5 core targets with these 12 compounds. Results showed that the highest score of docking were DRD2-Jujuboside B, BDNFJujuboside B, COMT-Jujuboside B, SLC6A4-Jujuboside B, HTR1A-Anemarsaponin B_qt (Fig. 8) (Table 7) (Additional file 11).

There were many studies 19-21 indicated that cannabinoid receptor 1 (CNR1) and mitogen-activated protein kinase 3 (MAPK3) played the key role in the pathophysiological mechanism of anxiety. Therefore, we executed molecular docking of compounds with molecular weight $₫ 500 \mathrm{Da}$ in SZRP employing AutoDock Vina. Results showed there were the biggest affinity between CNR1 and Isomangiferin, MAPK3 and n-methylasimilobine (Fig. 8) (Table 7) (Additional file 11).

\section{Cell experiments for validating binding affinities of compounds and targets}

According to the results of molecular docking, the mean absolute value of the combined score of the three subtypes of CNR1 (5tgz, 5xra, 5xr8) with compounds was greater. And based on compounds being purchased and reported or not, we selected five compounds to verified the activity of targeting on CNR1 by experiments. These compounds were Liquiritigenin, Isomangiferin, Kaempferol, Emodin and Chrysophanol. The calcium Flux assay was used to detect the activation of these 5 compounds on CNR1 (CHO-K1/CNR1/Ga15 cell). The results showed the $\mathrm{EC}_{50}$ of positive compound CP-55940 targeting on CNR1 was $49.44 \mu \mathrm{M}$. The $\mathrm{EC}_{50}$ of Isomangiferin, Liquiritigenin, Kaempferol, Chrysophanol and Emodin activating on CNR1 were $5.271 \times 10^{-5} \mathrm{M}, 5.686 \times 10^{-5} \mathrm{M}, 5.228 \times 10^{-5} \mathrm{M}, 1.383 \times 10^{-4} \mathrm{M}$ and $5.437 \times 10^{-5} \mathrm{M}$, respectively (Fig. 9) (Additional file 12). Chrysophanol and Kaempferol had the maximum effective rate of $81 \%$ and $68 \%$.

\section{Discussion}

In this study, we found that 545 chemical constituents in herbs of SZRP might target on 1050 biomolecules, and play a role in the treatment of brain disease, mood disorder, etc. The effects of SZRP on anti-anxiety may be via regulating membrane potential and synaptic transmission, etc., which involve in 117 biomolecules, such as DRD2, BDNF, COMT, SLC6A4, HTR1A etc. Especially, we found that Chrysophanol, Kaempferol and Emodin activated CNR1 with $\mathrm{EC}_{50} 1.383 \times 10^{-4} \mathrm{M}, 5.228 \times 10^{-5} \mathrm{M}$ and $5.686 \times 10^{-5} \mathrm{M}$ respectively. 
Endocannabinoid is found in the neuro-immune systems of both animals and humans. Cannabinoid receptors are considered to be key regulators of anxiety, neurodegenerative diseases and other diseases 22. Cannabinoid receptors are divided into CNR1 and CNR2, which are involved in pain perception, stress suppression, memory, motor function regulation, cognition and emotional response 23-25. A large number of pharmacological studies have shown that CNR1 acted as an important regulator of anxietylike behavior. For example, in the elevated plus-maze and the light/dark avoidance task, the complete CNR1 null-mutant mice showed an anxiogenic-like phenotype, in contrast, the conditional mutant mice lacking CNR1 expression specifically in cortical glutamatergic neurons failed to show a similar phenotype 26. In addition, study showed that exploration was increased when CNR1 receptor was deleted in cortical and striatal GABAergic neurons, however, deletion of CNR1 receptor from cortical glutamatergic neurons caused a decreased exploration in these task 27. And in mice lacking the CNR1 receptor either in cortical glutamatergic or in GABAergic neurons, CNR1 receptors expressed in cortical glutamatergic neurons favors novelty seeking, whereas CNR1-dependent control of inhibitory GABAergic neurons favors behavioral inhibition by novelty-induced behavioral inhibition test 28. Thus, CNR1 on cortical glutamatergic and GABAergic neurons exert opposing on anxiety-like behavior, but this function seems to operate within specific limits of neuronal activity 29. Numerous pharmacological studies support the view of bidirectional regulation of anxiety behavior by CNR1. Interestingly, experimental studies have shown that anti-anxiolytic-like effects in the low dose of cannabinoids via the CNR1 receptor on cortical glutamatergic terminals, on the contrary, the CNR1 receptor on the GABAergic terminals induce an anxiogenic-like effect under a high-dose treatment 30. Experimental studies have also found, while a mild activation of CNR1 receptors in the prefrontal cortex ventral hippocampus attenuates anxiety and a slight CNR1 receptor stimulation in the amygdala results in an anxiogenic-like response 31. Taken together, although the regulation of anxiety behavior by CNR1 showed bidirectional in different brain regions and different types of neurons, the complete CNR1 null-mutant body showed anxiety. Therefore, it might be effective or therapeutic for anxiety to activate CNR1 moderately. In this study, we found Chrysophanol, Kaempferol and Emodin in SZRP activated CNR1 with $\mathrm{EC}_{50} 138.3,52.28$ and $56.86 \mu \mathrm{M}$ respectively.

Chrysophanol (molecular weight $254.2 \mathrm{~g} / \mathrm{mol}_{1} \mathrm{C}_{15} \mathrm{H}_{10} \mathrm{O}_{4}$ ) belonged to anthraquinones and was yellow powder and soluble in methanol and ethanol. In a study of seahorses, compound Chrysophanol has been found that it relieved the anxious behavior of mice induced by chronic unpredictable mild stress (CUMS), which were correlated with reducing IL-1 $\beta$ and reactive oxygen species (ROS), increasing neurotransmitter (NE, DA, 5-HT) concentrations and BDNF expression 32.

Flavonoids Kaempferol (molecular weight $286.2 \mathrm{~g} / \mathrm{mol}, \mathrm{C}_{15} \mathrm{H}_{10} \mathrm{O}_{6}$ ) was yellow powder and slightly soluble in water, soluble in hot ethanol, ether or alkali solution. Some studies indicated Kaempferol had effects on anxiety. Kaempferol can enhanced number of entries and time spent in the open arm inEPM test, which demonstrated anxiolytic activity 33. Kaempferol from the leaves of Apocynum venetum possesses anxiolytic activities in the EPM test in mice, and benzodiazepine antagonist flumazenil was partially antagonized the anxiolytic activity of kaempferol 34. Kaempferol from Tagetes genus performed anxiolytic effects in open-field, exploration cylinder, hole-board task, and this anxiolytic effect was 
significantly inhibited in the presence of the receptor antagonist 5-HT1A (WAY 1006355) 35. Using light/dark model and hole-board experiment to evaluated whether Kaempferol has anti-anxiety activity in mice model, results showed Kaempferol had effects on anxiety and at a dose up to $2000 \mathrm{mg} / \mathrm{kg}$ body weight showed no mortality 36 .

Anthraquinones Emodin (molecular weight $270.2 \mathrm{~g} / \mathrm{mol}, \mathrm{C}_{15} \mathrm{H}_{10} \mathrm{O}_{5}$ ) was yellow powder and soluble in ethanol, slightly soluble in ether, chloroform, benzene, insoluble in water. It has the role of antiinflammatory $37-39$, neuroprotective 40 and cardiovascular protective ${ }^{41,42}$. No reports were found about Emodin effecting on anxiety.

Based on protein-protein interaction network related to Suanzaoren prescription for anti-anxiety, we found some possible important biomolecules regulated by Suanzaoren prescription, such as DRD2, BDNF, COMT, SLC6A4, HTR1A, etc.

Dopamine receptor D2 (DRD2) belonged to the G protein-coupled receptors. DRD2 receptor is the common drug target for anti-psychotics 43 . Some studies showed DRD2 played a role in anxiety disorders, for example, anxiety disorders in children and adolescents 44 . The expression of DRD2 significantly increased in the anxiety mouse models established with constrain stress and social isolation 45. And nicotine-dependent smokers enhanced prefrontal DRD2, might influence vulnerability for anxiety disorders 46.

The brain derived neurotrophic factor (BDNF) is a critical contributor to neuronal growth, development, learning, and memory 47. BDNF expression associated with the pathophysiology of anxiety ${ }^{48,49}$. Clinical studies have revealed that GAD has been showed lowered plasma BDNF levels 50-52. BDNF also may play a central role in the pathogenesis of bipolar disorder 53. In our previous experimental study of SZRP, it was found that SZRP can improve the BDNF mRNA expression level in insomnia rat, thus improving neuronal damage, improve the symptoms of insomnia ${ }^{54,55}$. And SZRP can also up-regulate the expression of BDNF genes, and has an anti-depressant effect 56 .

Catechol-0-methyltransferase (COMT) inactive catecholamine neurotransmitters and catechol hormones, also shortens the biological half-lives of certain neuroactive drugs. Some findings demonstrated that COMT Val (158) Met polymorphism is associated with risk of GAD via reduced resting parasympathetic nervous control 57 and COMT SNPs also modulated the association between antenatal maternal anxiety 58. Experimental research found that COMT KO mice showed increased anxiety 59.

Sodium-dependent serotonin transporter (SLC6A4) is sodium-dependent serotonin transporter, whose primary function in the central nervous system involves the regulation of serotonergic signaling. SLC6A4 are associated with vulnerability to affective disorders. Clinical case analysis found that, the serotonin transporter gene SLC6A4 involved in the etiology of anxiety-related ${ }^{60,61}$. It was found in some experimental studies that serotonin transporter expression was associated positively with anxiety-like behavior ${ }^{62,63}$. 
5-hydroxytryptamine receptor $1 \mathrm{~A} \otimes \mathrm{HTR} 1 \mathrm{~A} \rrbracket$ was G-protein coupled receptor for 5-hydroxytryptamine (serotonin) and also function as a receptor for various drugs and psychoactive substances. HTR1A played a role in the regulation of dopamine and 5-hydroxytryptamine levels in the brain, and thereby affect neural activity, mood and behavior. The study showed that mice with 5-HT1AR gene knockout showed anxiety-like behavior 64. 5-HT1AR neurotransmission-related dysfunction has been associated with several psychiatric disorders, including anxiety 65 , in 5-HT neurons to repress HTR1A expression and drive adult anxiety-like behaviors 66 . SZRP can reduce time for voluntary activities and regulate the expression of 5-HT1AR in the hippocampus of rats, thus improving the sleep state of sleep-deprived rats 67-69.

Mitogen-activated protein kinase $3 \triangle \mathrm{MAPK} 3 \rrbracket$ is mitogen-activated protein kinase 3 , which acts as an essential component of the MAP kinase signal transduction pathway, and thus suppress cancer cells 70 . MAPK3 is also associated with mental illness, such as autism spectrum disorder 71 .

Triterpenoids Jujuboside $\mathrm{B}$ (molecular weight $1045.2 \mathrm{~g} / \mathrm{mol}, \mathrm{C}_{52} \mathrm{H}_{84} \mathrm{O}_{21}$ ) was easier to get into the blood and white crystalline powder, soluble in methanol, almost insoluble in ether. It has activities of inhibiting leukemia cell growth 72, anti-inflammatory 73 , ameliorate cognitive deficiency 74 , antiplatelet 75 and etc. Based on molecular docking, we found Jujuboside B in SZRP might target on DRD2, BDNF, COMT and SLC6A4.

Steroids Anemarsaponin B_qt (molecular weight $416.71 \mathrm{~g} / \mathrm{mol}, \mathrm{C}_{45} \mathrm{H}_{74} \mathrm{O}_{18}$ ) was easy to cross the bloodbrain barrier 17 and white powder, soluble in benzene, pyridine and chloroform, soluble in ethanol, methanol and acetone, slightly soluble in water. Pharmacological action of Anemarsaponin B_qt has not been reported. But the pharmacological effects of similar compounds have been reported.

Anemarsaponin BII mainly increased bone formation 76, Anemarsaponin B has anti-inflammatory 77 and inhibit platelet aggregation 78. In this study, we found Anemarsaponin B_qt in SZRP might target HTR1A.

Alkaloids n-methylasimilobine (molecular weight $281.3 \mathrm{~g} / \mathrm{mol}_{1} \mathrm{C}_{18} \mathrm{H}_{19} \mathrm{NO}_{2}$ ) was yellow powder and soluble in methanol, ethanol, DMSO and other organic solvents. It has antioxidative activity 79 , significant cytotoxic activities against carcinoma cell line 80 and inhibition of platelet aggregation 81 . Based on molecular docking, this study showed that n-methylasimilobine in SZRP might target on MAPK3.

In this study, biological process enrichment analysis of potential target for shared target indicated that the regulation of membrane potential and GABAergic synaptic transmission might be some mechanism of SZRP anti-anxiety. Regulation of membrane potential refers to the regulation of the potential difference between the two sides of the membrane. Regulation of membrane potential have been implicated in diverse pathophysiological processes, including mood regulation etc. According to experimental research reports, the medial habenula ( an epithalamic structure implicated in anxiety-like behavior), neurons are capable of firing transient, high-frequency action potential bursts mediated by T-type channels in C57BL/6J mice 82. But an impaired maintenance of long-term potentiation in the hippocampus and 
display enhanced anxiety-like behavior of Np65 KO mice 83. However, membrane potential increased and anxiety-like behavior were significantly relieved after the treatment of G-1 (the GPER agonist) 84. Similarly, postsynaptic membrane potential and GABAergic synaptic transmission are closely related to anxiety disorders. Mechanisms underlying the anxiolytic effects of metformin by up-regulated the surface expression of GABAA receptors and increased miniature inhibitory postsynaptic currents 85 . The study showed that in EPM model rats treated by SZRP, the OE and OT were significantly increased, and the content of GABA was extremely significantly elevated 86 and the mRNA expression level of GABAA receptor was up-regulated 14. After being treated by SZRP, the content of GABA in hypothalamus, brainstem and brain tissue increased in insomnia model rat with chronic sleep deprivation, insomnia model rat with liver and blood deficiency and elderly insomnia model rats 87-90. In insomnia rats with fatigue model, SZRP could down-regulate the levels of GABA in the cerebral cortex and hypothalamus 91. It is not reported that SZRP may regulate membrane potential in anxiety.

In additional, pathways enrichment analysis of potential target for shared target of SARP and anxiety suggested that pathway nicotine addiction, retinol metabolism, serotonergic synapse might be regulated by SZRP. Nicotine addiction that means nicotine dependence and addictive behavior. However, nicotine addiction and nicotine withdrawal can lead to anxiety-like behavior 92-94. Experimental research report that activation of the neuronal PPARgamma 区the receptor for the thiazolidinedione class of medications $₫$ prevents the expression of signs of nicotine withdrawal 95 and increased expression of CRF (Corticotropin-Releasing Factor) and CRF-receptors in certain areas of the brain after the development of intraperitoneal nicotine administrations in rats 96 . Interestingly, the hippocampus seems to be closely linked to nicotine 97-99 and is also known to be associated with anxiety 100-102. Retinol (vitamin A囚metabolism refers to retinol comes out of the liver and binds to retinol-binding proteins, which are transported to various tissues of the body through the blood for normal metabolism. Retinol metabolism is involved in oxidative processes 103, 104 in body, and is also associated with anxiety. Retinol induced anxiety-like behavior in adult rats chronically subjected to vitamin A supplementation 104, 105. Serotonin (5-HT) is an important neurotransmitter, which is high in the cerebral cortex and synapses. Serotonin in the brain controls emotional regulation, cognitive function and other functions. Neurotransmitters such as serotonin and synapses are known to play an important role in the development of anxiety 106 . It was found that the integrity of serotonergic synapses and anxiety related phenotype in mice 107. It was worth noting that only a few studies had considered relationship between pre-and post-synaptic serotonergic transmission. Anxiety caused by serotonergic synapse was due to an imbalance of serotonergic synapse regulated mRNAs expression, such as $5-\mathrm{HTR}_{2 \mathrm{~A}}$ is upregulated 108, 5$\mathrm{HTR}_{1 \mathrm{~A}}$ down-regulated 109.

It was found that SZRP exhibited binding affinity for serotonin receptors on the basis of Ultraperformance liquid-chromatography/electrospray-ionization synapt high-definition mass spectrometry (UPLC/ESI-SYNAPT-HDMS) combined with pattern recognition approaches 110. A clinical study reported that the treatment of SZRP increased the serum 5-HT content in patients with liver blood deficiency insomnia 111. In the EPM rat model of anxiety, the content of 5-HIAA in the hippocampus and thymus of 
SZRP group was significantly higher than that of the model group $11,16,86$. And the content of $5-\mathrm{HT}$ and 5-HIAA in serum, hypothalamus, hippocampus, cerebral cortex and brain stem increased in model rats of insomnia and sleep deprivation rats treated by SZRP 68, 89-91, 112-115. And in Alzheimer's disease rats induced by D-galactose and A $31-42$, serum $5-H T$ concentration in SZRP group was significantly higher than that in model group 116. Other experimental reported that chronic stress induced depression in rat, compared with model group, SZRP significantly increased the content of 5-HT in both hippocampus and cerebral cortex 117. The administration of SZRP regulated the expression of $5-\mathrm{HT}_{1 \mathrm{~A}} \mathrm{R}$ and $5-\mathrm{HT}_{2 \mathrm{~A}} \mathrm{R}$ in hippocampus, hypothalamus, and thymus of rat with chronic sleep deprivation $67 \cdot 69$. However, there was no reports about the treatment of SZRP regulating serotonergic synapse in anxiety. While SZRP is involved in the therapy of anxiety by way of the nicotine addiction, retinol metabolism, serotonergic synapse, which we first discovered.

\section{Conclusions}

In this study, the therapeutic components and mechanism of SZRP for anxiety disorders were investigated by using network pharmacology combined with experimental verification. The main findings were as follows: (1) the 525 compounds and 1050 potential targets in SZRP were collected, and SZRP might be used to treat brain disease, mood disorder, endocrine system disease, bipolar disorder, etc. (2) the 249 potential targets of anxiety were obtained, and 117 possible targets of SZRP against anxiety were found, for example, DRD2, BDNF, COMT, SLC6A4, HTR1A and other biological molecules. (3) the 117 potential targets involved in some biological process, including of regulation of membrane potential, and synaptic transmission, GABAergic, etc. (4) the 117 potential targets played roles in many pathways, such as nicotine addiction, retinol metabolism, GABAergic and serotonergic synapse. (5) based on molecular docking, the biggest affinity were found between Jujuboside B and DRD2, BDNF, COMT, SLC6A4, Anemarsaponin B_qt and HTR1A, n-methylasimilobine and MAPK3. (6) Chrysophanol, Kaempferol and Emodin have activation effects on CNR1 with $\mathrm{EC}_{50} 138.3 \mu \mathrm{M}, 52.28 \mu \mathrm{M}$ and $56.86 \mu \mathrm{M}$ respectively. However, there are limitations to this study. The ingredients in SZRP and anxiety-related genes may not be global or full. We did not verify all the affinity between compounds and targets employing experiments. In conclusion, this study provided theoretical basis and clues for the study on the pharmacological mechanism of SZRP against anxiety.

\section{Abbreviations}




\begin{tabular}{ll} 
SZRP & Suanzaoren prescription \\
\hline CNR1 & cannabinoid receptor 1 \\
\hline DSM-IV & Diagnostic and Statistical Manual of Mental Disorders \\
\hline TCM & traditional Chinese medicine \\
\hline GAD & generalized anxiety disorder \\
\hline HAMA & Hamilton Anxiety Scale \\
\hline SAS & Self-Rating Anxiety Scale \\
\hline OE & open arm entry \\
\hline OT & open arm time \\
\hline EPM & elevated plus maze \\
\hline B-EP & B-endorphin \\
\hline ACTH & adreno-cortico-tropic-hormone \\
\hline H-C network & herb-compound network \\
\hline C-T network & compound-targets network \\
\hline CUMS & chronic unpredictable mild stress \\
\hline ROS & reactive oxygen species \\
\hline DRD2 & Dopamine receptor D2 \\
\hline BDNF & brain derived neurotrophic factor \\
\hline COMT & Catechol-O-methyltransferase \\
\hline SLC6A4 & Sodium-dependent serotonin transporter \\
\hline HTR1A & 5-hydroxytryptamine receptor 1A \\
\hline BP & Biological Process \\
\hline
\end{tabular}

\section{Declarations}

Acknowledgments The National Natural Science Foundation of China (No.81473191, 81974555) financially supported this research work.

Conflicts of interest The authors declare that there is no conflict for publication of this paper.

\section{References}

1. L. N. Ravindran and M. B. Stein, J Clin Psychiatry, 2010, 71, 839-854. 
2. J. Alonso, Z. R. Liu, S. Evans-Lacko, E. Sadikova, N. Sampson, S. Chatterji, J. Abdulmalik, S. AguilarGaxiola, A. Al-Hamzawi, L. H. Andrade, R. Bruffaerts, G. Cardoso, A. Cia, S. Florescu, G. de Girolamo, O. Gureje, J. M. Haro, Y. L. He, P. de Jonge, E. G. Karam, N. Kawakami, V. Kovess-Masfety, S. Lee, D. Levinson, M. E. Medina-Mora, F. Navarro-Mateu, B. E. Pennell, M. Piazza, J. Posada-Villa, M. ten Have, Z. Zarkov, R. C. Kessler, G. Thornicroft and W. W. M. H. Survey, Depression and Anxiety, 2018, 35, 195-208.

3. Y. Q. Huang, Y. Wang, H. Wang, Z. R. Liu, X. Yu, J. Yan, Y. Q. Yu, C. G. Kou, X. F. Xu, J. Lu, Z. Z. Wang, S. L. He, Y. F. Xu, Y. L. He, T. Li, W. J. Guo, H. J. Tian, G. M. Xu, X. D. Xu, Y. J. Ma, L. H. Wang, L. M. Wang, Y. P. Yan, B. Wang, S. Y. Xiao, L. Zhou, L. J. Li, L. W. Tan, T. T. Zhang, C. Ma, Q. Li, H. Ding, H. C. Geng, F. J. Jia, J. F. Shi, S. L. Wang, N. Zhang, X. B. Du, X. D. Du and Y. Wu, Lancet Psychiatry, 2019, 6, 211-224.

4. H. Mohler, Cell Tissue Res, 2006, 326, 505-516.

5. M. Uusi-Oukari and E. R. Korpi, Pharmacol Rev, 2010, 62, 97-135.

6. L. Menif, B. Oueslati, A. Maamri, W. Melki and H. Zalila, J Ethn Subst Abuse, 2019, DOI: 10.1080/15332640.2019.1589611, 1-13.

7. S. Bu and G. Ma, China Practical Medicine $\llbracket i n$ Chinese】, 2012, 7, 189-190.

8. H. Petursson, Addiction, 1994, 89, 1455-1459.

9. X. Jiang, H. Xu, H. Zhou and Q. Guo, The Northern PharmaceuticalDin Chinesel, 2020, 17, 83-84.

10. Y. Gu, Z. Xu, H. Chen and I. Xiong, Modern Traditional Chinese Medicinelin Chinese/, 2015, 35, 21-23.

11. X. Zhang, F. Tian, W. Zou, Y. Zhang and S. Zhang, Western Traditional Chinese MedicineIin Chinesel, 2017, 30, 11-13.

12. S. Wang and M. Xie, Journal of Beijing University of Traditional Chinese MedicineПin Chinese】, 2012, 35, 117-120+124.

13. X. Wang and M. Xie, Experimental Traditional Medical Formulaelin Chinese[, 2004, 35-37.

14. X. Wang, Beijing University of Chinese Medicine『in Chinese $₫, 2004$.

15. J. Wang, H. Li, J. Liu, X. Zhu and Z. Xie, Journal of Sichuan of Traditional Chinese Medicinelin Chinesel, 2009, 27, 21-22.

16. X. Wang and M. Xie, Experimental Traditional Medical Formulae』in Chinese『, 2005, 49-52.

17. Z. Sun, Shandong University of Traditional Chinese Medicine囚in Chinese®, 2013.

18. X. Wang and M. Xie, Journal of Beijing University of Traditional Chinese Medicine $\amalg$ in Chinese $\square, 2004$, 49-51. 
19. E.-B. Christian, L. C. F, R.-P. Javier, Z. A. M, M.-R. Jaime, P.-M. Hernán, R.-G. Gonzalo, I.-V. Patricio, T. R. A and P.-M. C. David, 2015, 348.

20. I. M. R, C. Resat, L. Jie, G. Grzegorz, S. Gergö, P. Henry, I. S. R, D. Jeffrey, L. Yong-Sok, S. P. J and K. George, 2015, 88.

21. B. Lutz, G. Marsicano, R. Maldonado and C. J. Hillard, Nat Rev Neurosci, 2015, 16, 705-718.

22. L. Khurana, K. Mackie, D. Piomelli and D. A. Kendall, Neuropharmacology, 2017, 124, 3-12.

23. A. K. Andrade, B. Renda and J. E. Murray, Pharmacol Biochem Behav, 2019, 180, 60-73.

24. M. A. Djeungoue-Petga and E. Hebert-Chatelain, Bioessays, 2017, 39.

25. S. C. Azad, J. Kurz, G. Marsicano, B. Lutz, W. Zieglgansberger and G. Rammes, Learn Mem, 2008, 15, 143-152.

26. W. Jacob, A. Yassouridis, G. Marsicano, K. Monory, B. Lutz and C. T. Wotjak, Genes Brain Behav, 2009, 8, 685-698.

27. M. Haring, N. Kaiser, K. Monory and B. Lutz, PLoS One, 2011, 6, e26617.

28. P. Lafenetre, F. Chaouloff and G. Marsicano, Neuropharmacology, 2009, 57, 715-721.

29. I. Katona and T. F. Freund, Annu Rev Neurosci, 2012, 35, 529-558.

30. A. A. Rey, M. Purrio, M. P. Viveros and B. Lutz, Neuropsychopharmacology, 2012, 37, 2624-2634.

31. T. Rubino, C. Guidali, D. Vigano, N. Realini, M. Valenti, P. Massi and D. Parolaro, Neuropharmacology, $2008,54,151-160$.

32. K. Li, L. Yan, Y. Zhang, Z. Yang, C. Zhang, Y. Li, A. V. Kalueff, W. Li and C. Song, J Ethnopharmacol, 2020, 250, 112487.

33. H. Ahmad, K. Rauf, W. Zada, M. McCarthy, G. Abbas, F. Anwar and A. J. Shah, Molecules, 2020, 25.

34. O. Grundmann, J. Nakajima, K. Kamata, S. Seo and V. Butterweck, Phytomedicine, 2009, 16, 295-302.

35. G. Perez-Ortega, G. E. Angeles-Lopez, A. Argueta-Villamar and M. E. Gonzalez-Trujano, Biomed Pharmacother, 2017, 93, 383-390.

36. V. Gupta, R. Sharma, P. Bansal and G. Kaur, Ayu, 2018, 39, 21-28.

37. M. R. de Oliveira, I. C. C. de Souza and F. B. Brasil, Neurochem Res, 2020, DOI: 10.1007/s11064-02003181-1. 
38. Z. Gao, J. Sui, R. Fan, W. Qu, X. Dong and D. Sun, Drug Des Devel Ther, 2020, 14, 1971-1982.

39. R. Guo, Y. Li, M. Han, J. Liu and Y. Sun, Int Immunopharmacol, 2020, 85, 106626.

40. S. W. Leung, J. H. Lai, J. C. Wu, Y. R. Tsai, Y. H. Chen, S. J. Kang, Y. H. Chiang, C. F. Chang and K. Y. Chen, Int J Mol Sci, 2020, 21.

41. L. W. Evans, A. Bender, L. Burnett, L. Godoy, Y. Shen, D. Staten, T. Zhou, J. E. Angermann and B. S. Ferguson, J Nutr Biochem, 2020, 79, 108339.

42. J. Gao, K. Zhang, Y. Wang, R. Guo, H. Liu, C. Jia, X. Sun, C. Wu, W. Wang, J. Du and J. Chen, Pharmacol Res, 2020, 155, 104739.

43. T. Schwalbe, J. Kaindl, H. Hubner and P. Gmeiner, Bioorg Med Chem, 2017, 25, 5084-5094.

44. T. T. Fraporti, V. Contini, L. Tovo-Rodrigues, M. Recamonde-Mendoza, D. L. Rovaris, L. A. Rohde, M. H. Hutz, A. Salatino-Oliveira and J. P. Genro, Prog Neuropsychopharmacol Biol Psychiatry, 2019, 93, 214-220.

45. H. G. Chuang, N. H. A. Aziz, J. H. Wong, M. Mustapha, J. M. Abdullah, Z. Idris, Z. Abdullah, A. Alrafiah and S. Muthuraju, Eur Neuropsychopharmacol, 2020, DOI: 10.1016/j.euroneuro.2019.12.121.

46. A. Kobiella, S. Vollstadt-Klein, M. Buhler, C. Graf, H. G. Buchholz, N. Bernow, I. Y. Yakushev, C. Landvogt, M. Schreckenberger, G. Grunder, P. Bartenstein, C. Fehr and M. N. Smolka, Hum Brain Mapp, 2010, 31, 716-726.

47. T. Dembo, J. M. Braz, K. A. Hamel, J. A. Kuhn and A. I. Basbaum, eNeuro, 2018, 5.

48. H. J. Kang, K. O. Kim, J. W. Kim, S. W. Kim, M. S. Park, H. R. Kim, M. G. Shin, K. H. Cho and J. M. Kim, Int J Geriatr Psychiatry, 2019, 34, 1706-1714.

49. Z. Zou, J. Qiu, Y. Huang, J. Wang, W. Min and B. Zhou, Psychol Health Med, 2019, 24, 505-511.

50. R. Dutt, N. Shankar, S. Srivastava, A. Yadav and R. S. Ahmed, Int J Psychiatry Clin Pract, 2020, 24, 135142.

51. Y. Wang, H. Zhang, Y. Li, Z. Wang, Q. Fan, S. Yu, Z. Lin and Z. Xiao, J Affect Disord, 2015, 186, 7-12.

52. H. A. Chang, W. H. Fang, Y. P. Liu, N. S. Tzeng, J. F. Shyu, F. J. Wan, S. Y. Huang, T. C. Chang and C. C. Chang, J Abnorm Psychol, 2020, 129, 237-247.

53. G. M. Mandolini, M. Lazzaretti, A. Pigoni, G. Delvecchio, J. C. Soares and P. Brambilla, J Affect Disord, $2019,243,552-558$.

54. H. Wang, K. Luo and J. Wu, Chinese Journal of Experimental Traditional Medical Formulaelin Chinesel, 2012, 18, 235-239. 
55. H. Wang, K. Luo and Y. Zhao, Lishizhen Medicine and Materia Medica Research in Chinese】, 2013, 24, 1898-1900.

56. X. Tian, N. Hu, L. Song, X. Yan and W. Cheng, Journal of Traditional Chinese Medicine』in Chinese】, 2011, 39, 30-32.

57. H. A. Chang, W. H. Fang, F. J. Wan, N. S. Tzeng, Y. P. Liu, J. F. Shyu, S. Y. Huang, T. C. Chang and C. C. Chang, Psychoneuroendocrinology, 2019, 106, 57-64.

58. A. Qiu, T. A. Tuan, M. L. Ong, Y. Li, H. Chen, A. Rifkin-Graboi, B. F. Broekman, K. Kwek, S. M. Saw, Y. S. Chong, P. D. Gluckman, M. V. Fortier, J. D. Holbrook and M. J. Meaney, Am J Psychiatry, 2015, 172, 163172.

59. L. Desbonnet, O. Tighe, M. Karayiorgou, J. A. Gogos, J. L. Waddington and C. M. O'Tuathaigh, Behav Brain Res, 2012, 228, 351-358.

60. A. J. Forstner, S. Rambau, N. Friedrich, K. U. Ludwig, A. C. Bohmer, E. Mangold, A. Maaser, T. Hess, A. Kleiman, A. Bittner, M. M. Nothen, J. Becker, F. Geiser, J. Schumacher and R. Conrad, Psychiatr Genet, 2017, 27, 96-102.

61. D. J. Oathes, L. M. Hilt and J. B. Nitschke, PLoS One, 2015, 10, e0115820.

62. S. K. L. Quah, L. Mclver, A. C. Roberts and A. M. Santangelo, J Neurosci, 2020, 40, 4739-4749.

63. P. T. Hsueh, H. H. Wang, C. L. Liu, W. F. Ni, Y. L. Chen and J. K. Liu, PLoS One, 2017, 12, e0179970.

64. P. R. Albert, F. Vahid-Ansari and C. Luckhart, Front Behav Neurosci, 2014, 8, 199.

65. J. Staron, R. Bugno, A. S. Hogendorf and A. J. Bojarski, Expert Opin Ther Pat, 2018, 28, 679-689.

66. T. J. Philippe, F. Vahid-Ansari, Z. R. Donaldson, B. Le Francois, A. Zahrai, V. Turcotte-Cardin, M. Daigle, J. James, R. Hen, Z. Merali and P. R. Albert, Sci Rep, 2018, 8, 5788.

67. D. Xiao, J. Liu and B. Zhao, Western Journal of Traditional Chinese Medicine』in Chinese】, 2019, 32, 12-15.

68. J. Li, X. Guo, R. Zhang and Q. You, Lishizhen Medicine and Materia Medica Research \in Chinese】, $2017,28,530-532$.

69. R. Zhang, Hubei University of Traditional Chinese Medicine囚in Chinese囚, 2017.

70. T. T. Yu, C. Y. Wang and R. Tong, Eur Rev Med Pharmacol Sci, 2020, 24, 5267-5280.

71. S. M. Park, H. R. Park and J. H. Lee, Mol Cells, 2017, 40, 151-161. 
72. M. M. Jia, Y. Q. Li, K. Q. Xu, Y. Y. Zhang, S. M. Tan, Q. Zhang, J. Peng and X. J. Luo, Eur J Pharmacol, 2020, 876, 173041.

73. P. B. Ninave and S. D. Patil, Respir Physiol Neurobiol, 2019, 260, 28-36.

74. M. Zhang, C. Qian, Z. G. Zheng, F. Qian, Y. Wang, P. M. Thu, X. Zhang, Y. Zhou, L. Tu, Q. Liu, H. J. Li, H. Yang, P. Li and X. Xu, Theranostics, 2018, 8, 4262-4278.

75. E. J. Seo, S. Y. Lee, S. S. Kang and Y. S. Jung, Phytother Res, 2013, 27, 829-834.

76. L. Qin, T. Han, Q. Zhang, D. Cao, H. Nian, K. Rahman and H. Zheng, J Ethnopharmacol, 2008, 118, 271279.

77. J. Y. Kim, J. S. Shin, J. H. Ryu, S. Y. Kim, Y. W. Cho, J. H. Choi and K. T. Lee, Food Chem Toxicol, 2009, 47, 1610-1617.

78. J. X. Dong and G. Y. Han, Yao Xue Xue Bao, 1992, 27, 26-32.

79. C. M. Liu, C. L. Kao, H. M. Wu, W. J. Li, C. T. Huang, H. T. Li and C. Y. Chen, Molecules, 2014, 19, 1782917838.

80. X. H. Duan, L. Pei and J. Q. Jiang, Zhongguo Zhong Yao Za Zhi, 2013, 38, 4104-4108.

81. F. R. Chang, J. L. Wei, C. M. Teng and Y. C. Wu, Phytochemistry, 1998, 49, 2015-2018.

82. C. R. Vickstrom, X. Liu, Y. Zhang, L. Mu, T. J. Kelly, X. Yan, M. M. Hu, S. T. Snarrenberg and Q. S. Liu, eNeuro, 2020, 7.

83. H. Li, Y. Liu, X. Gao, L. Liu, S. Amuti, D. Wu, F. Jiang, L. Huang, G. Wang, J. Zeng, B. Ma and Q. Yuan, FEBS J, 2019, 286, 3401-3415.

84. J. Wang, R. Yu, Q. Q. Han, H. J. Huang, Y. L. Wang, H. Y. Li, H. M. Wang, X. R. Chen, S. L. Ma and J. Yu, Behav Brain Res, 2019, 359, 845-852.

85. J. Fan, D. Li, H. S. Chen, J. G. Huang, J. F. Xu, W. W. Zhu, J. G. Chen and F. Wang, Br J Pharmacol, 2019, 176, 297-316.

86. Y. Zhang, Shaanxi College of Traditional Chinese Medicine囚in Chinese区, 2014.

87. L. Lin, Hubei University of Traditional Chinese Medicine『in Chinese囚, 2014.

88. J. Zheng, K. Chen and C. Wu, Journal of Hubei University of Traditional Chinese Medicine『in Chinesel, 2020, 22, 9-12.

89. J. Guo and X. Yin, China Pharmacyロin Chinesel, 2016, 27, 3085-3087. 
90. L. Lin, Hubei University of Traditional Chinese Medicine『in Chinese囚, 2014.

91. L. Zhang, Guangzhou University of Chinese Medicine囚in Chinese囚, 2009.

92. L. C. Smith, M. Kallupi, L. Tieu, K. Shankar, A. Jaquish, J. Barr, Y. Su, N. Velarde, S. Sedighim, L. L. G. Carrette, M. Klodnicki, X. Sun, G. de Guglielmo and O. George, Neuropsychopharmacology, 2020, 45, 19091919.

93. A. Pisera-Fuster, M. P. Faillace and R. Bernabeu, Mol Neurobiol, 2020, 57, 1828-1846.

94. M. Casarrubea, C. Davies, M. Pierucci, R. Colangeli, G. Deidda, A. Santangelo, S. Aiello, G. Crescimanno and G. Di Giovanni, Prog Neuropsychopharmacol Biol Psychiatry, 2020, 105, 110131.

95. E. Domi, F. F. Caputi, P. Romualdi, A. Domi, G. Scuppa, S. Candeletti, A. Atkins, M. Heilig, G. Demopulos, G. Gaitanaris, R. Ciccocioppo and M. Ubaldi, J Neurosci, 2019, 39, 9864-9875.

96. L. Carboni, B. Romoli, S. T. Bate, P. Romualdi and M. Zoli, Drug Alcohol Depend, 2018, 189, 12-20.

97. J. K. Brynildsen, B. G. Lee, I. J. Perron, S. Jin, S. F. Kim and J. A. Blendy, Proc Natl Acad Sci U S A, 2018, $115,4282-4287$.

98. M. G. Kutlu, D. A. Connor, J. M. Tumolo, C. Cann, B. Garrett and T. J. Gould, Neuropharmacology, 2018, $141,192-200$.

99. M. L. Fisher, R. M. LeMalefant, L. Zhou, G. Huang and J. R. Turner, Neuropsychopharmacology, 2017, 42, 1599-1609.

100. M. Zhuang, Q. Lai, C. J. Yang, Y. H. Ma, B. M. Fan, Z. X. Bian, C. Y. Lin, J. Bai and G. Z. Zeng, Biochem Bioph Res Co, 2020, 525, 326-333.

101. V. Rytova, D. E. Ganella, D. Hawkes, R. A. D. Bathgate, S. Ma and A. L. Gundlach, Hippocampus, 2019, 29, 905-920.

102. S. Ahmadi and S. Khaledi, Metabolic Brain Disease, 2020, 35, 579-588.

103. F. Klamt, F. Dal-Pizzol, D. P. Gelain, R. S. Dalmolin, R. Birnfeld de Oliveira, M. Bastiani, F. Horn and J. C. Fonseca Moreira, Cell Biol Int, 2008, 32, 100-106.

104. M. R. de Oliveira and J. C. Moreira, Toxicol Lett, 2007, 173, 145-150.

105. M. R. de Oliveira, R. B. Silvestrin, E. S. T. Mello and J. C. Moreira, Neurotoxicology, 2007, 28, 11911199.

106. K. Kobayashi, Y. Mikahara, Y. Murata, D. Morita, S. Matsuura, E. Segi-Nishida and H. Suzuki, Iscience, 2020, 23. 
107. A. Bregin, M. Kaare, T. Jagomae, K. Karis, K. Singh, K. Laugus, J. Innos, E. Leidmaa, I. Heinla, T.

Visnapuu, E. M. Oja, K. Koiv, K. Lillevali, J. Harro, M. A. Philips and E. Vasar, Pharmacol Biochem Be, 2020, 198.

108. Y. Sun, W. Lu, K. X. Du and J. H. Wang, Journal of Neurophysiology, 2019, 122, 1002-1022.

109. J. Y. Yang, J. Y. Sun, Y. J. Lu, T. T. An, W. Lu and J. H. Wang, Psychopharmacology, 2020, 237, 30673093.

110. B. Yang, A. Zhang, H. Sun, W. Dong, G. Yan, T. Li and X. Wang, J Pharm Biomed Anal, 2012, 58, 113124.

111. S. Wang, S. Chen, Y. Chen, L. Liu, X. Li and X. Chen, Shanghai journal of traditional chinese medicine Din Chinesel, 2020, 54, 50-56.

112. B. Yang and M. Yue, Shanxi traditional Chinese medicinelin Chinese[, 2015, 36, 123-124.

113. X. Zhang, J. Sun and L. Bai, Pharmacology and clinic of traditional Chinese medicinelin Chinese】, 2014, 30, 8-11.

114. H. Xia, Journal of Zhejiang University of Traditional Chinese Medicine』in Chinese】, 2010, 34, 52-53.

115. X. Zhang, F. Tian, W. Zou, Y. Zhang and S. Zhang, Henan Traditional Chinese Medicine】in Chinese】, 2016, 36, 985-987.

116. H. Yuan, Wannan Medical College囚in Chinese囚, 2016.

117. X. Tian, L. Song, B. Ma and W. Cheng, Traditional Chinese medicine information $\square$ in Chinese $\square, 2014$, 31, 47-49.

\section{Tables}

Due to technical limitations, table 1-7 is only available as a download in the Supplemental Files section.

\section{Figures}




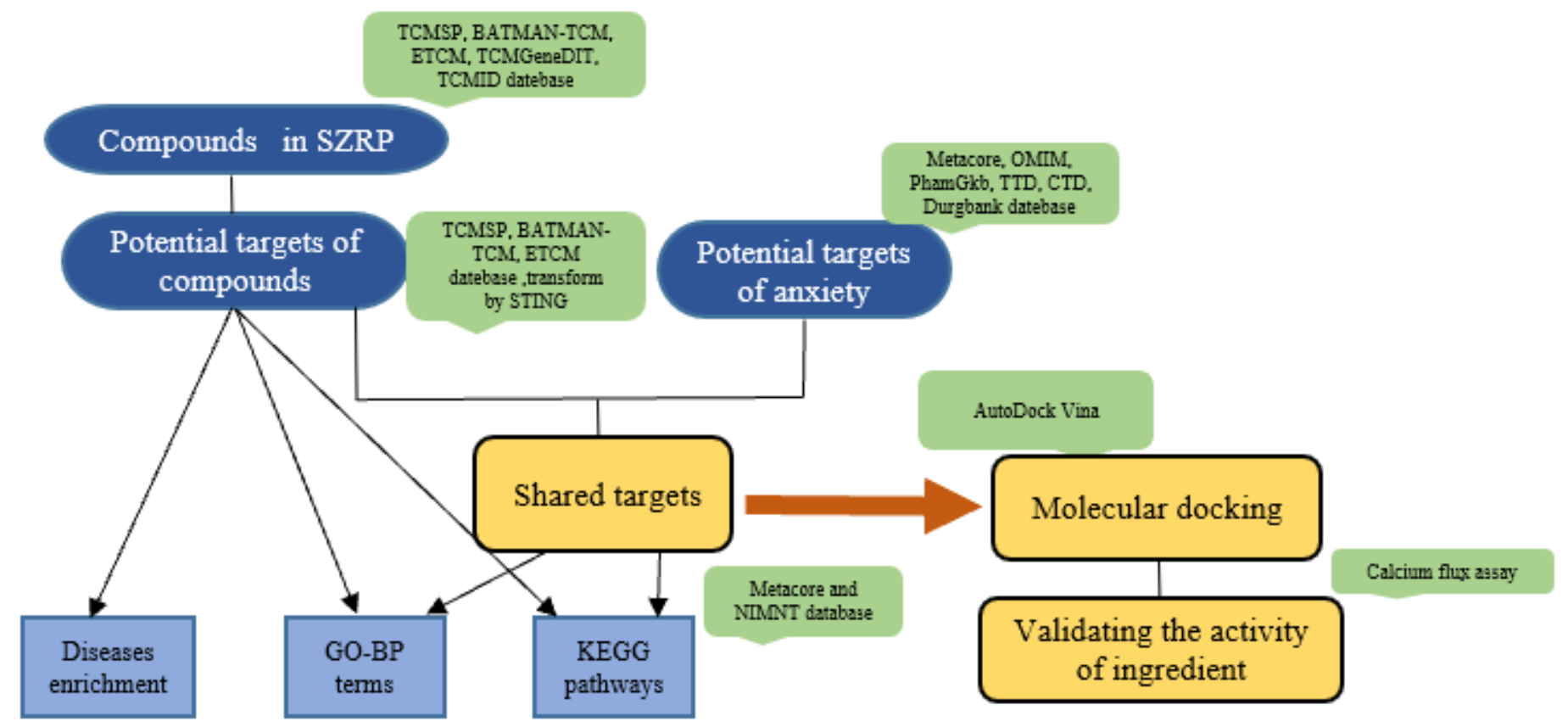

Figure 1

The workflow of network pharmacology approach to investigate the mechanisms of Suanzaoren prescription against anxiety. 


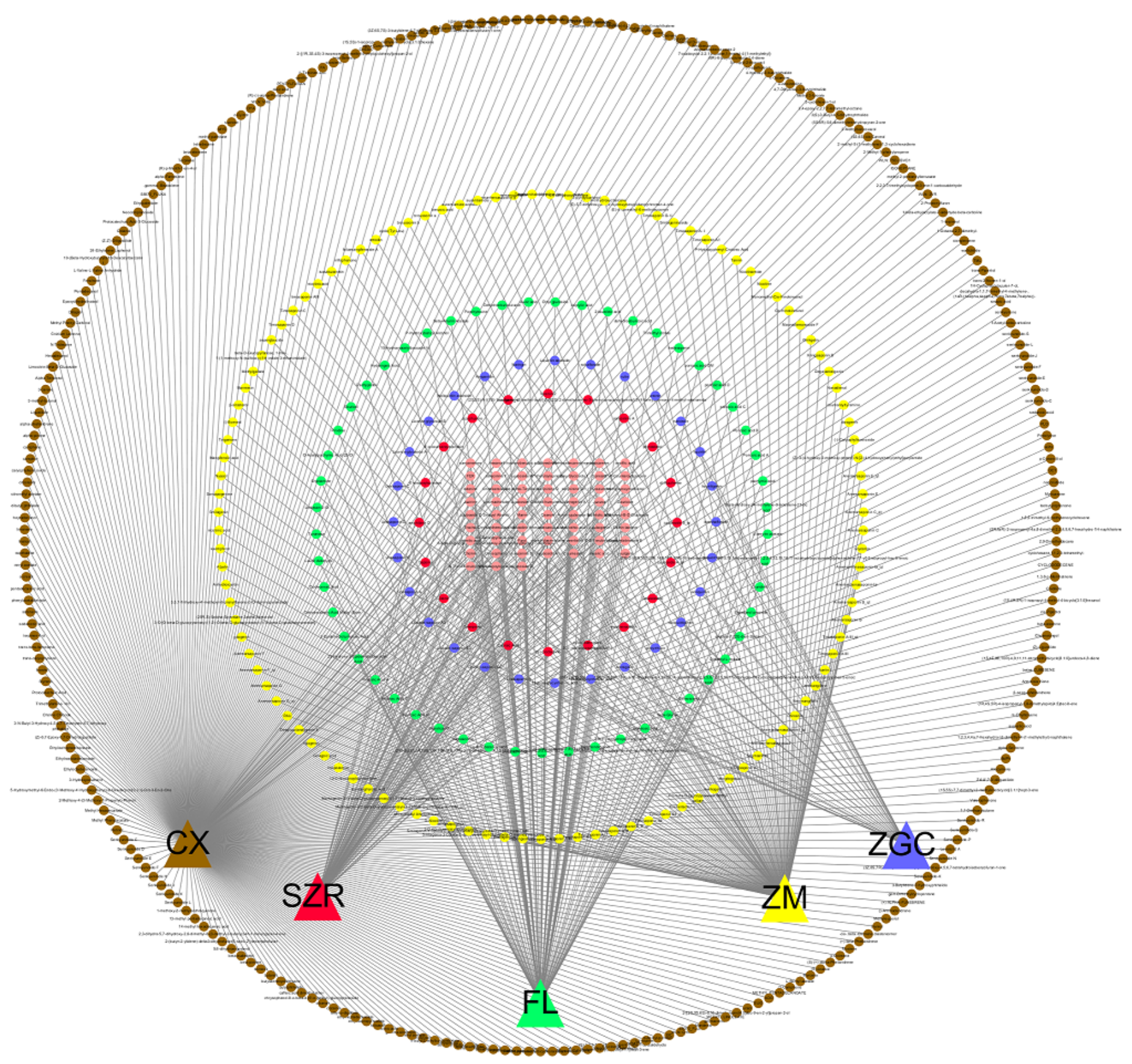

Figure 2

The network of compound and herb in Suanzaoren prescription. This network comprised 550 nodes and 610 edges (herb-compound). The triangle represents traditional Chinese medicine and the circle represents compounds. Suanzaoren (SZR) and its compounds are shown in red, Fuling (FL) and its compounds in green, Zhimu (ZM) and its compounds in yellow, Chuanxiong (CX) and its compounds in brown, zhigancao (ZGC) and its compounds in blue, pink nodes represent common compounds. 


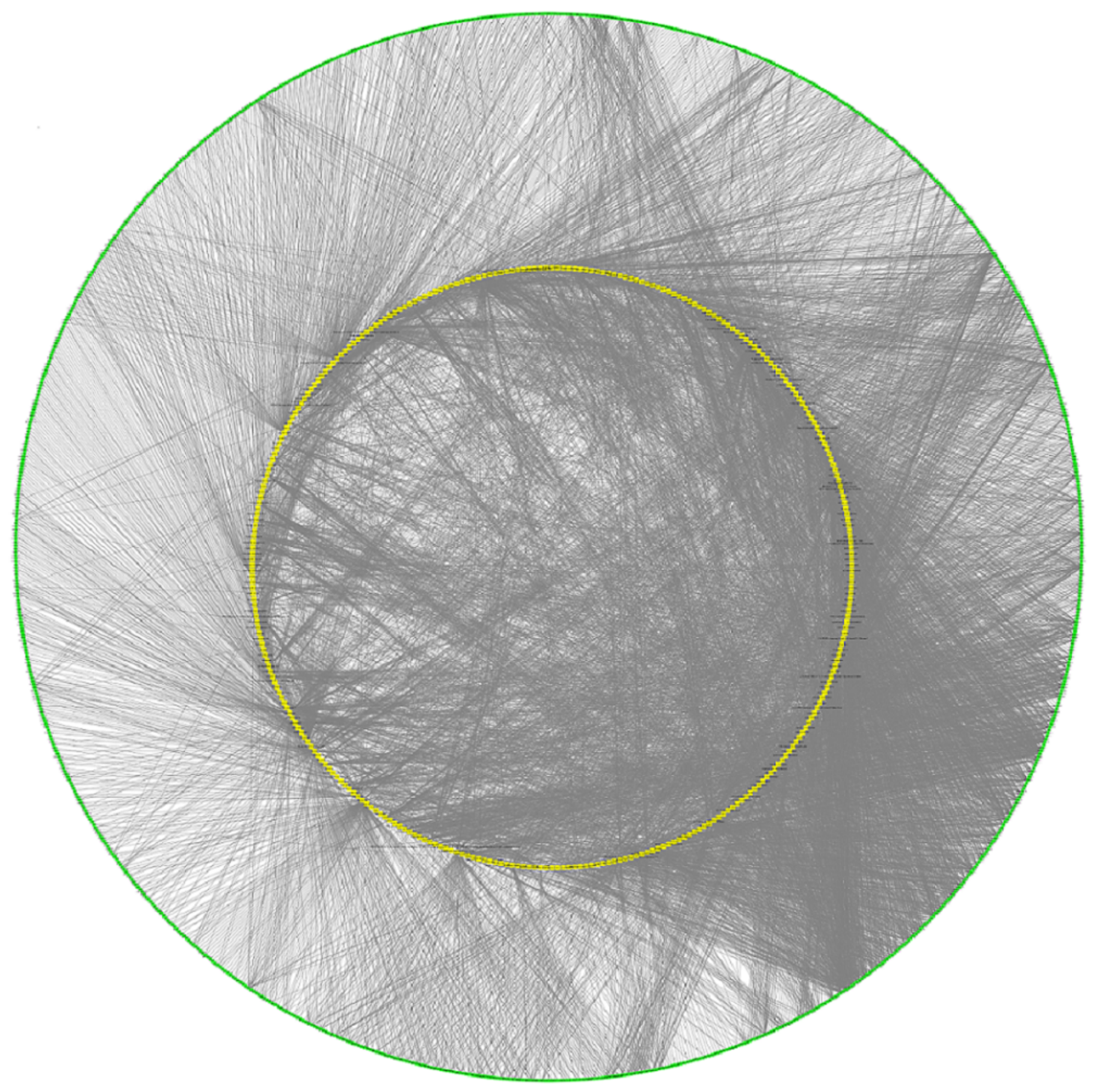

Figure 3

The network of compounds in herbs and potential targets in Suanzaoren prescription. Yellow node represents compounds, green node represents targets, and the line represents the interaction between compounds and potential targets. Isolated nodes are not shown in the diagram. 
$\mathbf{A}$

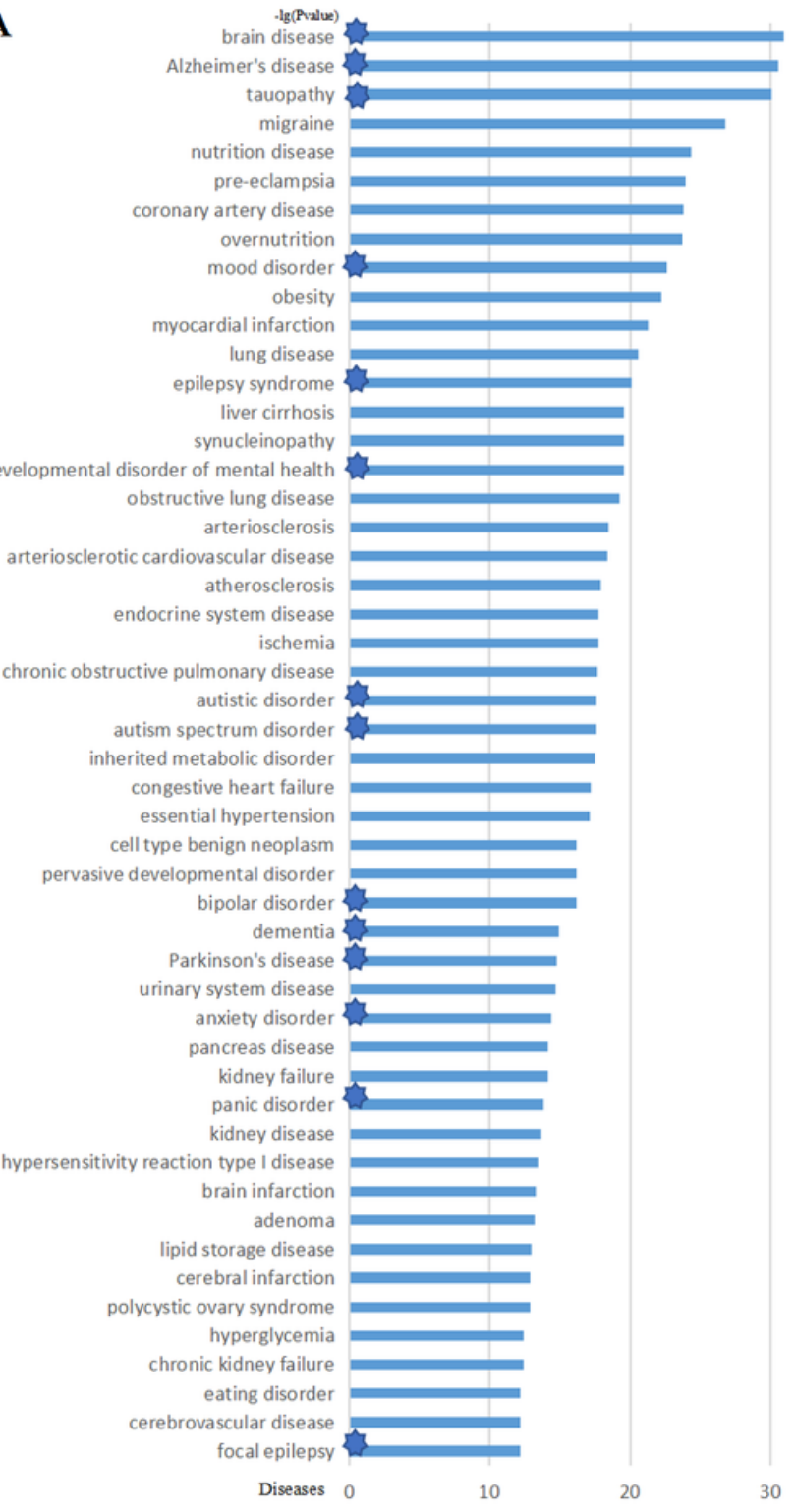

B Mental Disorders Nutritional and Metabolic Diseases Psychiatry and Psychology Metabolic Diseases

Movement Disorders Genetic Diseases, Inborn Congenital, Hereditary, and Neonatal Diseases an... Bipolar Disorder Bipolar and Related Disorders Basal Ganglia Diseases Central Nervous System Diseases Brain Diseases

Drug-Related Side Effects and Adverse Reactions Signs and Symptoms In-house Adverse Events Substance-Related Disorders Depressive Disorder Cognition Disorders Mood Disorders Pathologic Processes Depressive Disorder, Major Schizophrenia Spectrum and Other Psychotic... Schizophrenia Chorea Nervous System Disease Neurocognitive Disorders Dementia Neurodegenerative Diseases Endocrine System Diseases Huntington Disease Dyskinesias

Glucose Metabolism Disorders Lung Diseases, Obstructive Phenomena and Processes Diabetes Mellitus
Asthma

Respiratory Hypersensitivity Epileptic Syndromes Heredodegenerative Disorders, Nervous System Bronchial Diseases Hypersensitivity Immune System Diseases Nutrition Disorders Diabetes Mellitus, Type 2 Physiological Phenomena Alcoholism Musculoskeletal Diseases

Diseases 0
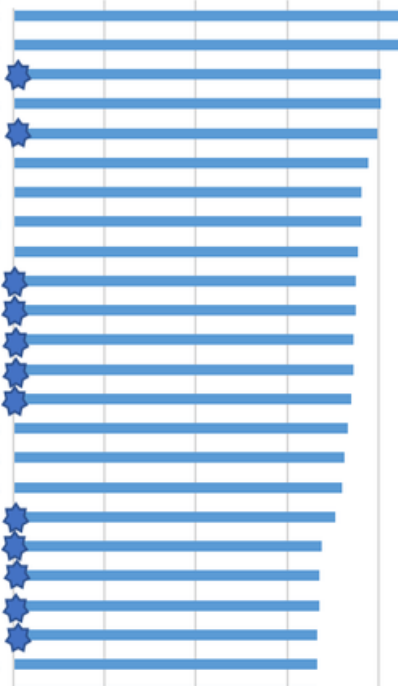

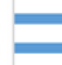
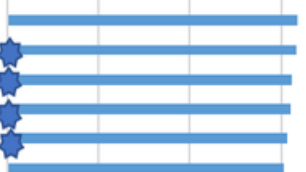

$=$
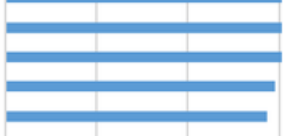

(1)
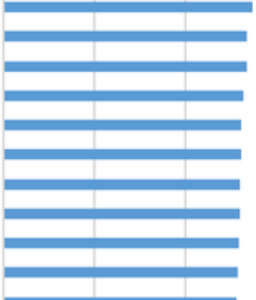

,

$\begin{array}{llll}50 & 100 & 150 & 200\end{array}$

\section{Figure 4}

The top 50 diseases enriched for the potential targets of the Suanzaoren prescription. A. Diseases enrichment from NIMNT; B. Diseases enrichment from MetaCore; Select the top 50 for visualization. Xaxis shows the -lg (P value) of the terms; $Y$-axis shows the different diseases $(P<0.001)$. * mean that the disease is related to nervous system or emotional disorder. 


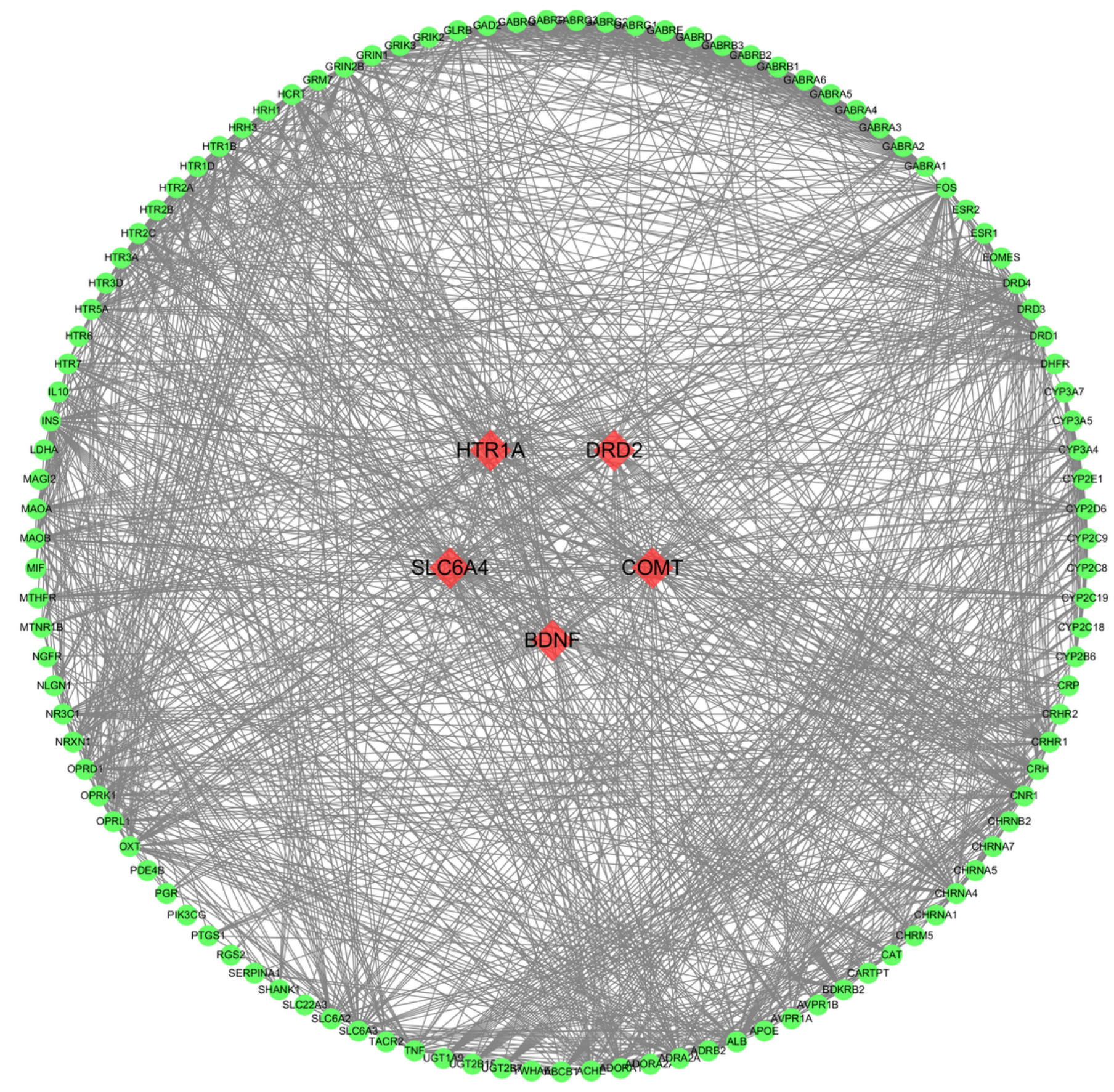

Figure 5

The protein-protein interaction network related to Suanzaoren prescription for anti-anxiety. This network had 116 nodes and 1336 edges (The one isolated hide disconnected node was not shown in this network). The red node is one with top 11 degree. 


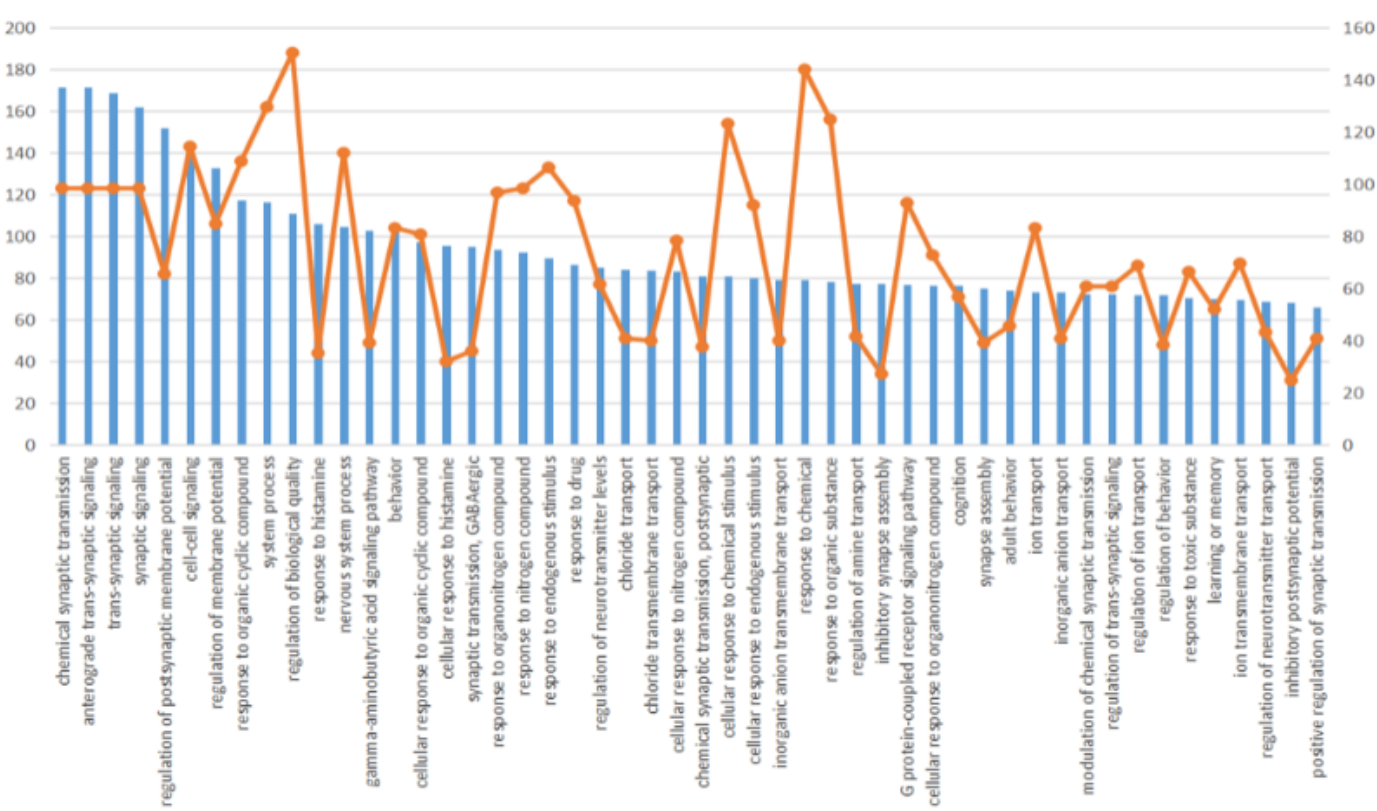

B

GO Processes

$-\lg ($ Pvalue $)$

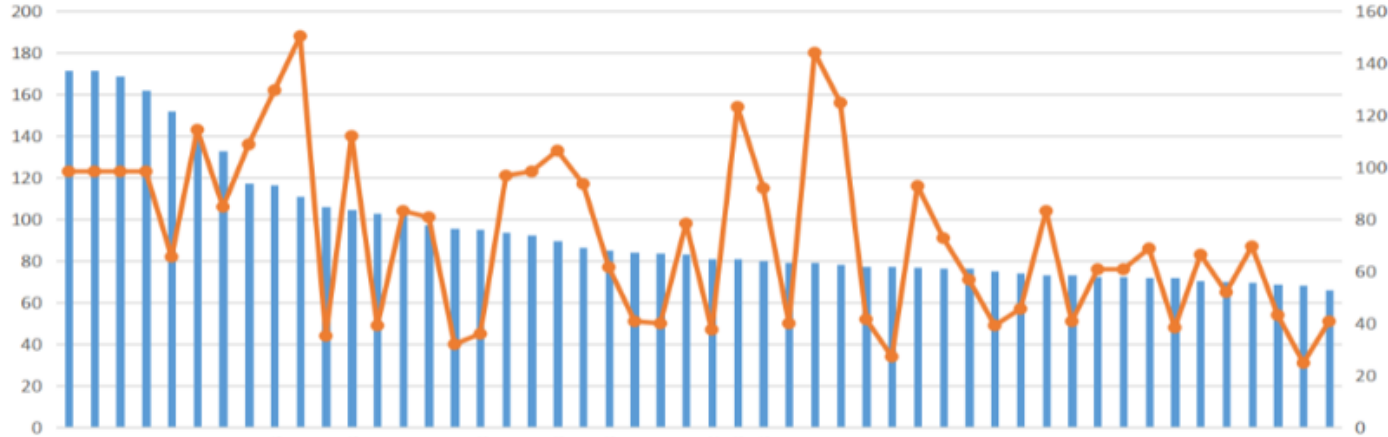

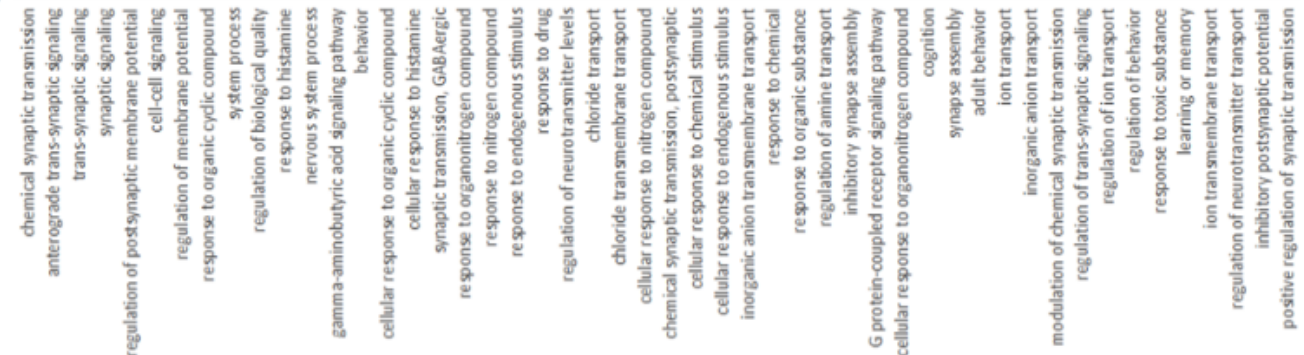

Figure 6

Top 50 biological process enrichment analysis of potential target for shared target. A. Biological processes enrichment from NIMNT; B. Biological process enrichment from MetaCore. X-axis shows the name of the biological processes, the left $Y$ axis shows gene number and the right $Y$ axis shows the - $\lg (P$ value $)$ of the terms $(P<0.001)$. 
$\mathbf{A}$

Pathway Analysis

-lg(Pvalue)

Neuroactive ligand-receptor interaction Nicotine addiction Serotonergic synapse Morphine addiction GABAergic synapse Drug metabolism - cytochrome P450 Retrograde endocannabinoid signaling

Chemical carcinogenesis Calcium signaling pathway

Taste transduction Retinol metabolism CAMP signaling pathway Cocaine addiction

Metabolism of xenobiotics by cytochrome P450 Steroid hormone biosynthesis Dopaminergic synapse Linoleic acid metabolism Amphetamine addiction Arachidonic acid metabolism Alcoholism $=$ Cholinergic synapse $=$ Phenylalanine metabolism Tyrosine metabolism cGMP-PKG signaling pathway Drug metabolism - other enzymes Glutamatergic synapse Ascorbate and aldarate metabolism = Regulation of lipolysis in adipocytes Gap junction = Bile secretion Antifolate resistance Rap1 signaling pathway = Pentose and glucuronate interconversions Inflammatory mediator regulation of TRP channels = Prolactin signaling pathway Porphyrin and chlorophyll metabolism = Tryptophan metabolism Type I diabetes mellitus One carbon pool by folate Histidine metabolism = Circadian entrainment Endocrine resistance = Pathway 0

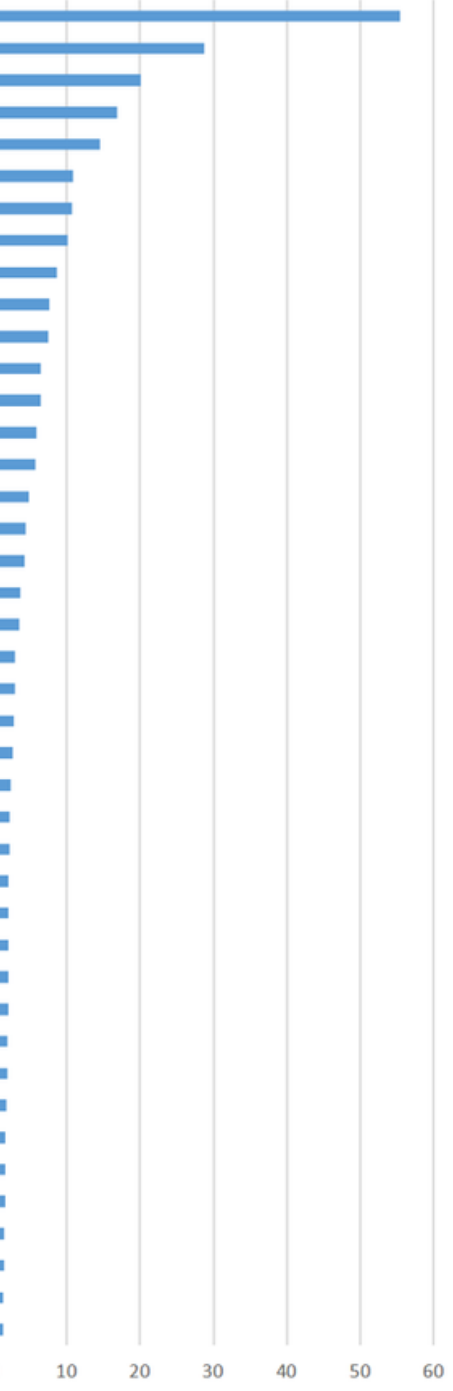

B

Pathway Analysis

Ig(Pvalue)

Tinnitus-associated changes in auditory pathway Protein folding and maturation Insulin processing PXR-mediated direct regulation of xenobiotic. CAR-mediated direct regulation of xenobiotic.. CAR-mediated direct regulation of xenobiotic. PXR-mediated direct regulation of xenobiotic. Nicotine signaling (general schema) Retinol metabolism Estradiol metabolism

Serotonin modulation of dopamine release in . Dysregulation of indirect glucose-induced inhibitio. CAR signaling via cross-talk / Human Version CAR signaling via cross-talk / Rodent version Nicotine signaling in dopaminergic neurons, Pt. 1 . Glucocorticoids-mediated inhibition of pro-. Neurophysiological process_Regulation of intrinsic. Huntingtin-depended transcription deregulation in..

possible role of neonatal nicotine exposure in.. Neurophysiological process GABA-A receptor life. Nociception_Expression and role of Nociceptin in. Nicotine signaling in glutamatergic neurons

Regulation of AKT(PKB)/ GSK3 beta cascade in... Cannabinoid receptor signaling in nicotine addiction Estrone metabolism

Gamma-Secretase regulation of neuronal cell. PR action in breast cancer: stimulation of cell...

Putative role of Estrogen receptor and Androgen.

Deregulation of PSD-95-dependent signaling in. TrkB signaling in Huntington's disease

NRSF-dependent transcription deregulation in.

Nociception Pro-nociceptive action of Nociceptin i... Degranulation of lung mast cells

Ubiquitin-proteasome system in Huntington's. Breast cancer (general schema)

Neurophysiological process_Constitutive and

Neurophysiological process_Glutamic acid. Neurophysiological process_Dopamine D2 recepto. mmune response PGE2 in immune and.

Neurophysiological process_HTR2A signaling in the.

$G$ protein-coupled receptors signaling in lung cancer

Protein folding and maturation_Posttranslational.. Huntington's disease (general schema)

Role of prenatal nicotine exposure in apoptosis an... Androstenedione and testosterone biosynthesis an.

Neurophysiological process_NMDA-dependent.. Androstenedione and testosterone biosynthesis an.. Activation of ACTH production in pituitary gland in.. Development_MAG-dependent inhibition of neurit.

Transcription_Assembly of RNA Polymerase II.. Nicotine signaling in GABAergic neurons

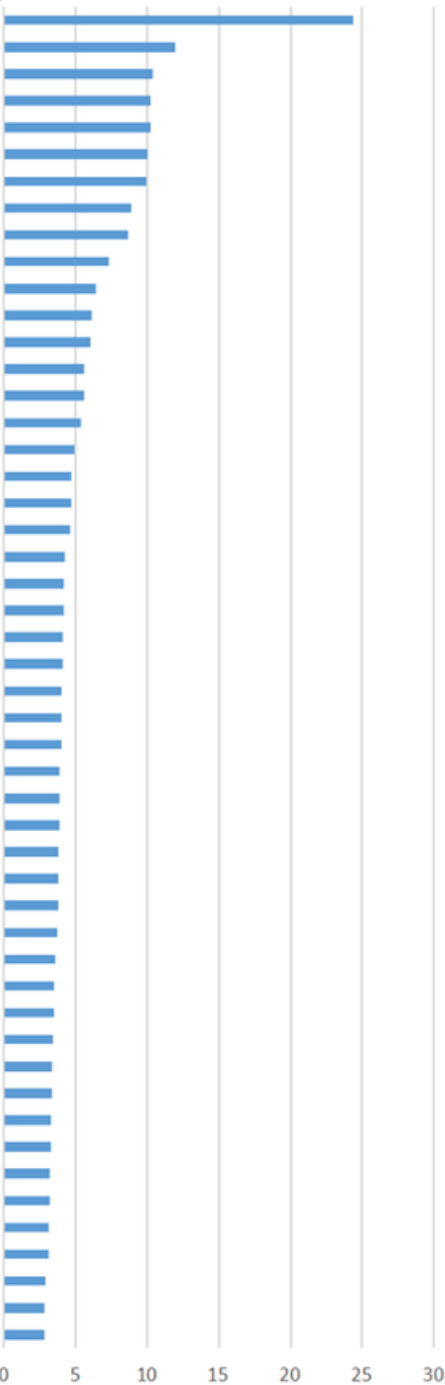

Figure 7

Top 50 pathways for gene target enrichment of potential target for shared target. A. Pathway enrichment come from NIMNT, $X$-axis shows the -lg ( $P$ value) of the terms, the left $Y$ axis shows the different pathway $(P<0.05)$. B. Pathway enrichment come from Metacore, $X$-axis shows the -lg ( $P$ value $)$ of the terms, the left $Y$ axis shows the different pathway $(P<0.01)$. 


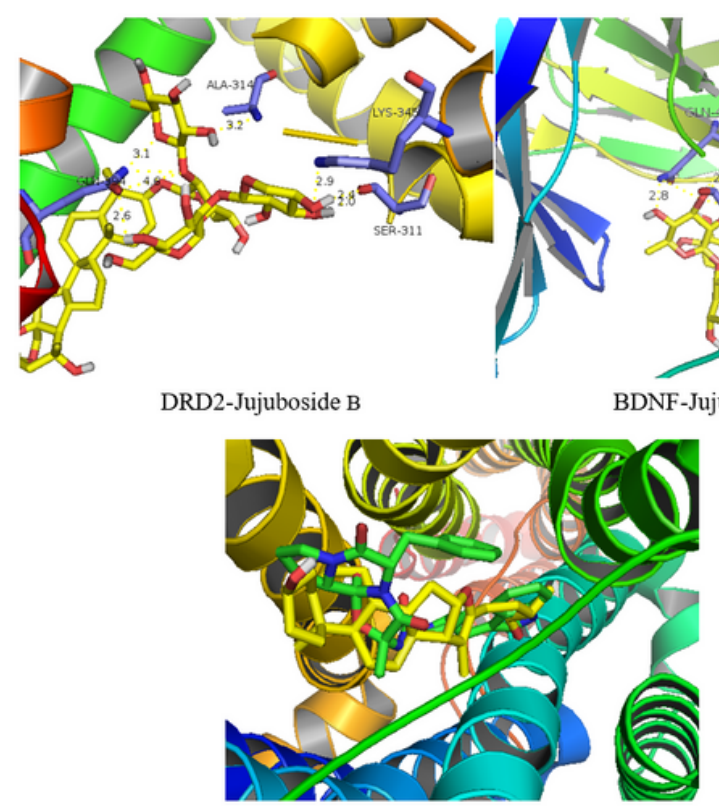

HTR1A-Anemarsaponin B_qt

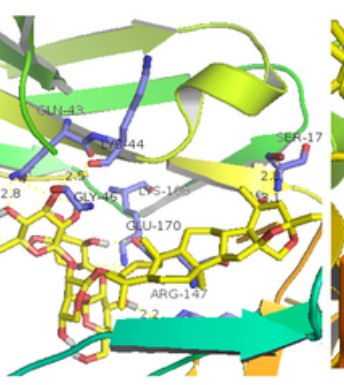

DNF-Jujuboside B

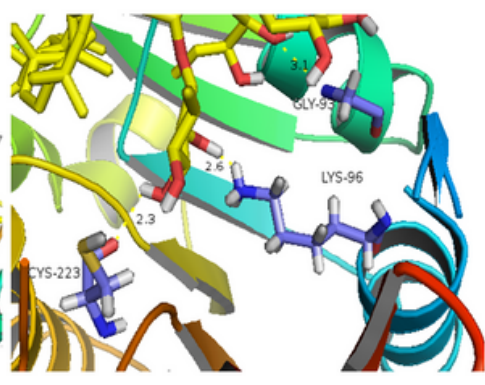

COMT-Jujuboside B

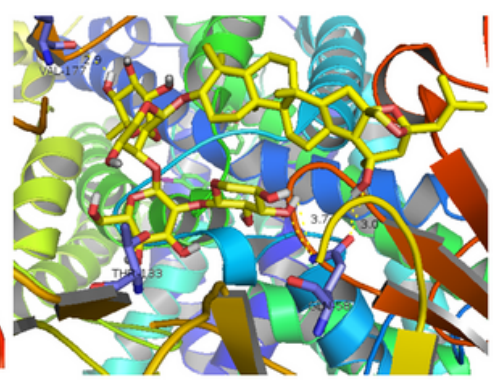

SLC6A4-Jujuboside B

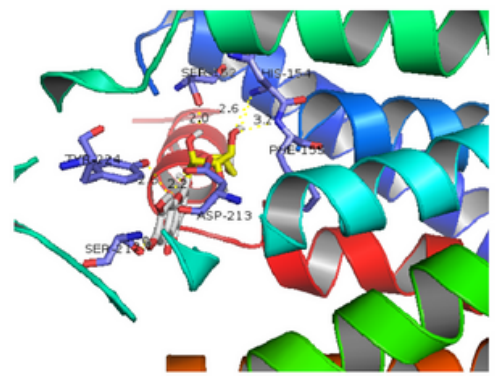

CNR1 (5xr8)-Isomangiferin

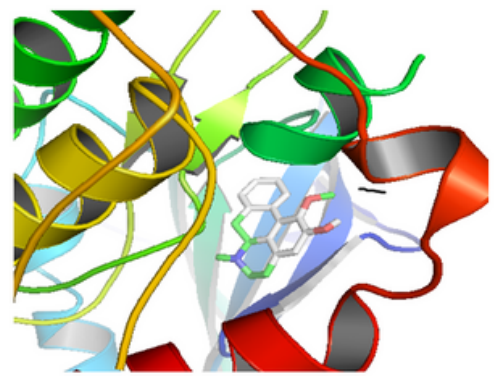

MAPK3-n-methylasimilobine

\section{Figure 8}

The molecular docking for compound in Suanzaoren prescription and core targets with related to antianxiety. * DRD2: Dopamine receptor D2; BDNF: brain derived neurotrophic factor; COMT: Catechol-Omethyltransferase; SLC6A4: sodium-dependent serotonin transporter; HTR1A: G-protein coupled receptor for 5-hydroxytryptamine; CNR1: Cannabinoid receptor 1;MAPK3: mitogen-activated protein kinase 3. (The dotted yellow line in the figure represents hydrogen bonds, and the number represents the bonding force between hydrogen bonds $\llbracket$. 
A

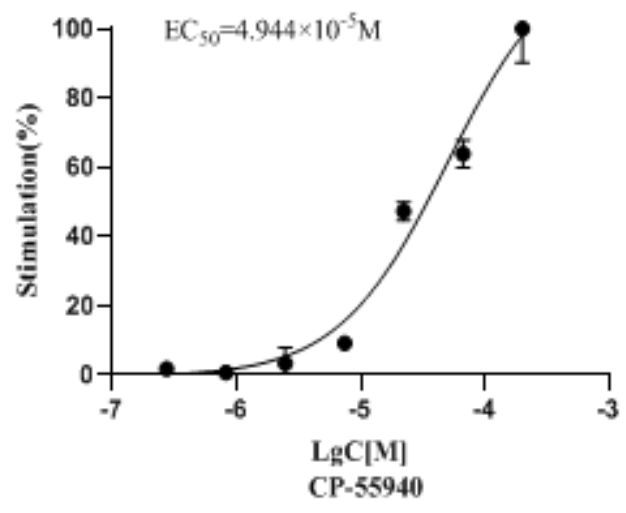

C

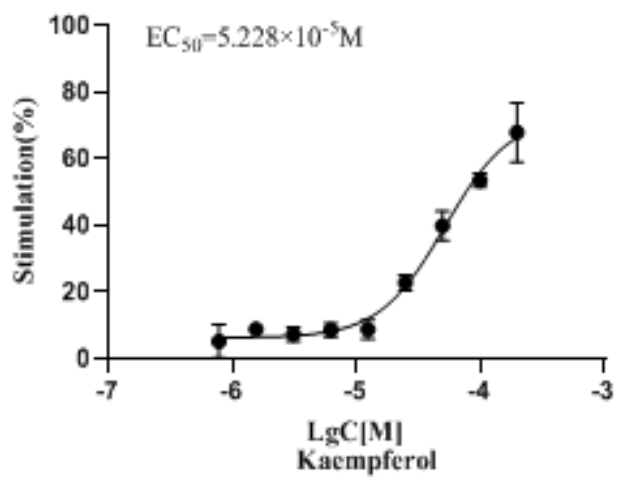

E

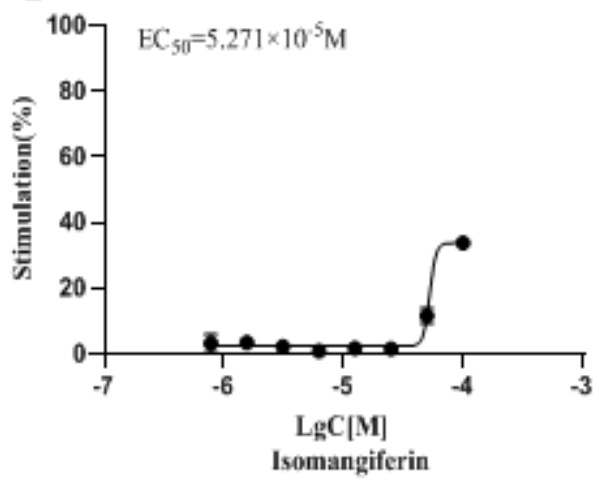

B

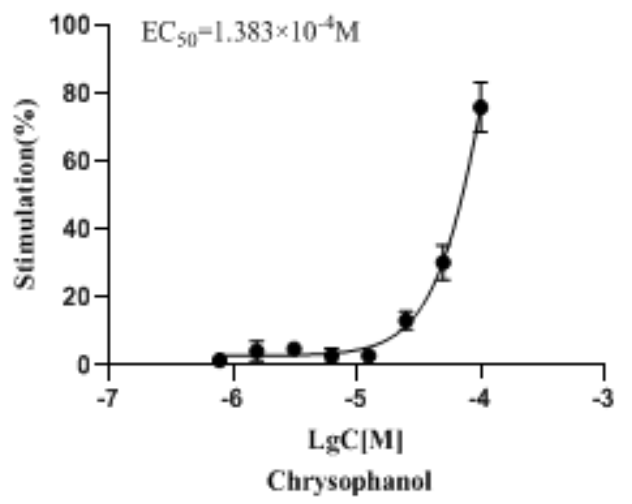

D

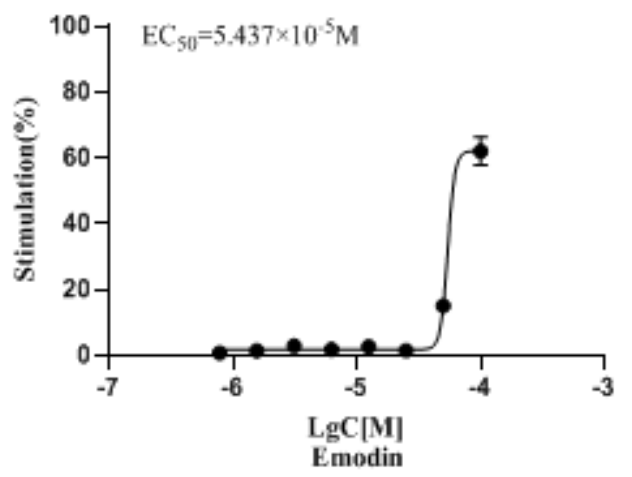

$\mathbf{F}$

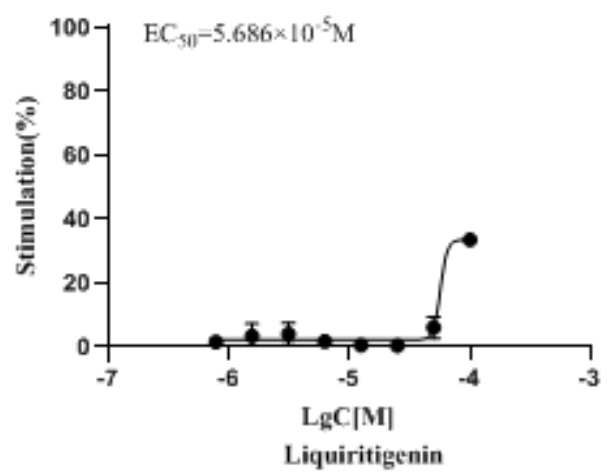

Figure 9

The EC50 of compound in Suanzaoren prescription against the target of Cannabinoid receptor 1.A. CP55940 - CNR1; B. Chrysophanol - CNR1; C. Kaempferol - CNR1; D. Emodin - CNR1; E. Isomangiferin - CNR1; F. Liquiritigenin - CNR1. Mean \pm S.E.M., $n=3$.

\section{Supplementary Files}

This is a list of supplementary files associated with this preprint. Click to download. 
- Supplementarymaterial.zip

- Table.pdf 